\title{
Convenient Methods for the Synthesis of Ferrocene-Carbohydrate Conjugates
}

\author{
Juan M. Casas-Solvas, ${ }^{\dagger}$ Antonio Vargas Berenguel ${ }^{*} \dagger$, Luis F. Capitán-Vallvey, ${ }^{\ddagger}$ \\ Francisco Santoyo-González ${ }^{*} \S$ \\ † Área de Química Orgánica, Universidad de Almería, 04120 Almería, Spain. \\ ${ }^{\S}$ Instituto de Biotecnología, Facultad de Ciencias, Universidad de Granada, 18071, Granada, Spain. \\ ${ }^{\star}$ Dpto. de Química Analítica, Facultad de Ciencias, Universidad de Granada, 18071, Granada, Spain. \\ avargas@ual.es; fsantoyo@ugr.es
}

\section{Supporting Information}

(35 Pages) 


\section{General Experimental}

TLC was performed on Merck Silica Gel $60 \mathrm{~F}_{254}$ aluminium sheets and developed by UV light and ethanolic sulfuric acid (5\% v/v). Flash column chromatography was performed on Silica Gel Merck (230-400 mesh, ASTM). Melting points were measured on a Gallenkamp and a Büchi B-450 melting point apparatuses and are uncorrected. Optical rotations were recorded on a Perkin Elmer 141 and an ADP 220 polarimeters at room temperature. IR spectra were recorded on a Mattson Satellite FTIR and a Mattson Genesis II FTIR. ${ }^{1} \mathrm{H}$ and ${ }^{13} \mathrm{C}$ NMR spectra were recorded on a Bruker Avance DPX $300 \mathrm{MHz}$ spectrometer. $\mathrm{CDCl}_{3}\left(\delta\left({ }^{1} \mathrm{H}\right)=7.26 \mathrm{ppm}, \delta\left({ }^{13} \mathrm{C}\right)=77.0 \mathrm{ppm}\right), \mathrm{CD}_{3} \mathrm{OD}\left(\delta\left({ }^{1} \mathrm{H}\right)=3.31 \mathrm{ppm}, \delta\left({ }^{13} \mathrm{C}\right)=49\right.$ ppm $), \operatorname{DMSO}_{6}\left(\delta\left({ }^{1} \mathrm{H}\right)=2.5 \mathrm{ppm}, \delta\left({ }^{13} \mathrm{C}\right)=39.5 \mathrm{ppm}\right)$ and HDO $\left(\delta\left({ }^{1} \mathrm{H}\right)=4.72 \mathrm{ppm}\right)$ were used as internal references. DEPT 135, COSY, HMQC, HMBC and selective 1D-TOCSY experiments were used for spectroscopic assignment. FAB mass spectra were recorded on a Fissons VG Autospec-Q spectrometer. MALDI-TOF mass spectra were recorded on a Bruker Autoflex spectrometer.

\section{Electrochemical Measurements}

Cyclic voltammetric (CV) and differential pulse voltammetric (DPV) experiments were carried out in sonicated, nitrogen-purged $\mathrm{H}_{2} \mathrm{O}$ (MilliQ $18.2 \mathrm{M} \Omega \mathrm{cm}$ ) solution with a Metrohm 663 VA-Stand instrument interfaced to an Autolab PSTAT10 potentiostat connected to a Pentium $133 \mathrm{MHz}$ personal computer running Eco Chimie B. V. GPES 4.9 software under Windows 95. The concentrations of the compounds were $0.5 \mathrm{mM}$ with $\mathrm{NaCl} 50 \mathrm{mM}$ as supporting electrolyte. A solution of $\mathrm{NaCl} 50 \mathrm{mM}$ was used as blank and its signal was subtracted from those of the compounds. Temperature of the solutions were measured with a digital thermometer Digi-sense $\mathbf{J}$ Thermocouple. The working electrode was glassy carbon, the counter electrode Platinum Ingold Swiss Made, and the reference electrode $\mathrm{Ag} / \mathrm{AgCl}, \mathrm{KCl} 3$ M. The working electrode surface was carefully polished with a basic $\mathrm{Al}_{2} \mathrm{O}_{3}$-water slurry, washed with

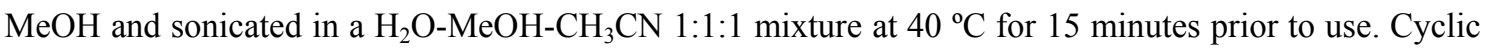
voltammograms were obtained measuring 5 scans at 6 sweep rates varying from $0.05 \mathrm{~V} / \mathrm{s}$ to $0.5 \mathrm{~V} / \mathrm{s}$. DPV experiments were 3 times measured with a scan rate of $0.01 \mathrm{~V} / \mathrm{s}$, a pulse height of $0.05 \mathrm{~V}$, and a duration of $0.05 \mathrm{~s}$. The diffusion coefficients were calculated by plotting $\mathrm{I}_{\mathrm{pc}}$ versus square root of $v$. The effective area of the electrode $\left(0.399 \pm 0.009 \mathrm{~cm}^{2}\right)$ was determined with the same method by using $\mathrm{Na}_{4} \mathrm{Fe}(\mathrm{CN})_{6}(1$ $\mathrm{mM}$ in water, $\mathrm{KCl} 100 \mathrm{mM}$ as supporting electrolyte, $\left.D_{0}=0.65 \times 10^{-5} \mathrm{~cm}^{2} / \mathrm{s}\right){ }^{1}$

\section{Synthesis and Data}

Ferrocenemethanol (1), 1,1'-bis(hydroxymethyl)ferrocene (8) and 1-ethynylferrocene (26) were purchased from Aldrich and used as received. Solvents were dried according to literature procedures. ${ }^{2}$

\footnotetext{
${ }^{1}$ Kuwana, T; Bublitz, D. E.; Hoh, G.; J. Am. Chem. Soc., 1960, 82, 5811.

2 Perrin, D. D.; Armarego, W. F. L. Purfication of Laboratory Chemicals, 3rd ed., Pergamon Press, Oxford, 1989.
} 
Glycosyl thiols ${ }^{3}$ (2-4), propargyl glycosides ${ }^{4}$ (20 and 21) and 2-azidoethyl glycosides ${ }^{5}$ (27 and 28) were prepared as reported.

General procedure for the synthesis of thioglycosylated ferrocenes 5-7 and 9-11. To a solution of ferrocene derivative $\mathbf{1}$ or $\mathbf{8}$ and glycosyl thiol 2-4 (1.1 equiv per hydroxyl group) in dry $\mathrm{CH}_{2} \mathrm{Cl}_{2}(10 \mathrm{~mL}$ ) was added trifluoroacetic acid (3 equiv per hydroxyl group). The mixture was stirred for $2 \mathrm{~h}$ at room temperature. The solvent was removed by evaporation under vacuum and the crude was purified by column chromatography to yield the corresponding thioglycosylated ferrocene.

(2,3,4,6-Tetra- $O$-acetyl- $\beta$-D-glucopyranosyl)thiomethylferrocene (5). Starting from 1 ( $25 \mathrm{mg}$, $0.116 \mathrm{mmol}$ ), column chromatography (EtOAc-Hexane 1:2) gave $\mathbf{5}(61 \mathrm{mg}, 94 \%)$ as a yellow solid: M. p. $141{ }^{\circ} \mathrm{C} ;[\alpha]_{\mathrm{D}}-30^{\circ}\left(\mathrm{c} 1, \mathrm{CHCl}_{3}\right)$; IR $\left(\mathrm{KBr}, \mathrm{cm}^{-1}\right): 2951,2901,2858,1735,1376,1229,1043 ;{ }^{1} \mathrm{H}-\mathrm{NMR}$ $\left(300 \mathrm{MHz}, \mathrm{CDCl}_{3}\right), \delta(\mathrm{ppm}): 5.19\left(\mathrm{t}, 1 \mathrm{H},{ }^{3} J=9.2 \mathrm{~Hz}, \mathrm{H}-3\right), 5.07\left(\mathrm{t}, 1 \mathrm{H},{ }^{3} J=10.7 \mathrm{~Hz}, \mathrm{H}-4\right), 5.04\left(\mathrm{t}, 1 \mathrm{H},{ }^{3} J\right.$ $=10.0 \mathrm{~Hz}, \mathrm{H}-2), 4.40\left(\mathrm{~d}, 1 \mathrm{H},{ }^{3} J_{1,2}=9.8 \mathrm{~Hz}, \mathrm{H}-1\right), 4.26\left(\mathrm{dd}, 1 \mathrm{H},{ }^{2} J_{6,6}=12.3 \mathrm{~Hz},{ }^{3} J_{5,6}=5.0 \mathrm{~Hz}, \mathrm{H}-6\right), 4.14$ (s, $5 \mathrm{H}, \mathrm{H}_{\mathrm{Cp}}$ ), 4.20 (bs, 2H, $\mathrm{H}_{\mathrm{Cp}}$ ), 4.12 (bs, 3H, $\mathrm{H}_{\mathrm{Cp}}, \mathrm{H}-6$ '), $3.74\left(\mathrm{~d}, 1 \mathrm{H},{ }^{2} J=13.1 \mathrm{~Hz}, \mathrm{CH}_{2} \mathrm{~S}\right), 3.67(\mathrm{~m}, 1 \mathrm{H}$, $\mathrm{H}-5$ ), 3.65 (d, $1 \mathrm{H},{ }^{2} \mathrm{~J}=12.9 \mathrm{~Hz}, \mathrm{CH}_{2} \mathrm{~S}$ ), 2.11 (s, 3H, $\mathrm{CH}_{3} \mathrm{CO}$ ), 2.02 (s, 6H, $\mathrm{CH}_{3} \mathrm{CO}$ ), 3.99 (s, 3H, $\mathrm{CH}_{3} \mathrm{CO}$ ); ${ }^{13} \mathrm{C}-\mathrm{NMR}\left(75 \mathrm{MHz}, \mathrm{CDCl}_{3}\right), \delta(\mathrm{ppm}): 169.4(\mathrm{CO}), 83.5\left(\mathrm{C}_{\mathrm{ipso}}\right), 82.5(\mathrm{C}-1), 75.8(\mathrm{C}-5), 73.9(\mathrm{C}-3), 69.8$ (C-2), $68.9\left(\mathrm{C}_{\mathrm{Cp}}\right), 68.8\left(\mathrm{C}_{\mathrm{Cp}^{p}}\right)$, 68.6, $68.4\left(\mathrm{C}_{\mathrm{Cp}}\right), 68.3(\mathrm{C}-4), 68.0\left(\mathrm{C}_{\mathrm{Cp}}\right), 62.2(\mathrm{C}-6), 29.5\left(\mathrm{CH}_{2} \mathrm{~S}\right), 20.8$, 20.7, $20.6\left(\mathrm{CH}_{3} \mathrm{CO}\right)$; HMRS (FAB + ): Calc. for $\mathrm{C}_{25} \mathrm{H}_{30} \mathrm{O}_{9} \mathrm{SFe} 562.0960$. Found $585.0858[\mathrm{M}+\mathrm{Na}]^{+}$.

(2,3,4,6-Tetra- $\boldsymbol{O}$-acetyl- $\alpha$-D-mannopyranosyl)thiomethylferrocene (6). Starting from 1 (50 $\mathrm{mg}, 0.231 \mathrm{mmol}$ ), column chromatography (EtOAc-Hexane 1:2) gave $6(117 \mathrm{mg}, 90 \%)$ as a yellow solid: M. p. $151^{\circ} \mathrm{C} ;[\alpha]_{\mathrm{D}}+78^{\circ}\left(\mathrm{c} 1, \mathrm{CHCl}_{3}\right)$; IR $\left(\mathrm{KBr}, \mathrm{cm}^{-1}\right): 2968,2950,1746,1370,1226,1054 ;{ }^{1} \mathrm{H}-\mathrm{NMR}$ $\left(300 \mathrm{MHz}, \mathrm{CDCl}_{3}\right), \delta(\mathrm{ppm}): 5.30$ (bs, $\left.1 \mathrm{H}, \mathrm{H}-2\right), 5.29$ (bd, $\left.1 \mathrm{H},{ }^{3} J=1.0 \mathrm{~Hz}, \mathrm{H}-4\right), 5.26\left(\mathrm{t}, 1 \mathrm{H},{ }^{3} J=3.1 \mathrm{~Hz}\right.$, H-3), 5.20 (bs, 1H, H-1), 4.38-4.34 (m, 1H, H-5), 4.32 (dd, 1H, $J=12.4$ Hz, $J=5.9$ Hz, H-6), 4.20 (d, $\left.1 \mathrm{H},{ }^{3} J=1.7 \mathrm{~Hz}, \mathrm{H}_{\mathrm{Cp}}\right), 4.18\left(\mathrm{~d}, 1 \mathrm{H},{ }^{3} J=1.7 \mathrm{~Hz}, \mathrm{H}_{\mathrm{Cp}}\right), 4.14-4.11\left(\mathrm{~m}, 2 \mathrm{H}, \mathrm{H}_{\mathrm{Cp}}\right), 4.13\left(\mathrm{~s}, 5 \mathrm{H}, \mathrm{H}_{\mathrm{Cp}}\right), 4.07$ (bd, $1 \mathrm{H},{ }^{2} J_{6,6},=12.4 \mathrm{~Hz}, \mathrm{H}-6$ ') $3.65\left(\mathrm{~d}, 1 \mathrm{H},{ }^{2} J=13.5 \mathrm{~Hz}, \mathrm{CH}_{2} \mathrm{~S}\right), 3.57\left(\mathrm{~d}, 1 \mathrm{H},{ }^{2} J=13.6 \mathrm{~Hz}, \mathrm{CH}_{2} \mathrm{~S}\right), 2.14(\mathrm{~s}$, $3 \mathrm{H}, \mathrm{CH}_{3} \mathrm{CO}$ ), 2.13 (s, 3H, $\mathrm{CH}_{3} \mathrm{CO}$ ), 2.04 (s, 3H, $\mathrm{CH}_{3} \mathrm{CO}$ ), 1.97 (s, 3H, $\mathrm{CH}_{3} \mathrm{CO}$ ); ${ }^{13} \mathrm{C}-\mathrm{NMR}(75 \mathrm{MHz}$, $\left.\mathrm{CDCl}_{3}\right), \delta(\mathrm{ppm}):$ 170.4, 169.7, 169.6, $169.5(\mathrm{CO}), 83.4\left(\mathrm{C}_{\mathrm{ipso}}\right), 81.7$ (C-1), $70.6(\mathrm{C}-2), 69.5(\mathrm{C}-3), 68.8$ (C-5), $68.7\left(\mathrm{C}_{\mathrm{Cp}_{\mathrm{p}}}\right), 68.6\left(\mathrm{C}_{\mathrm{Cp}^{\prime}}\right)$, 68.5, 68.3, $68.0\left(\mathrm{C}_{\mathrm{Cp}}\right), 66.2(\mathrm{C}-4), 62.3(\mathrm{C}-6), 30.7\left(\mathrm{CH}_{2} \mathrm{~S}\right), 20.8,20.6,20.5$, $20.4\left(\mathrm{CH}_{3} \mathrm{CO}\right)$; HMRS (FAB+): Calc. for $\mathrm{C}_{25} \mathrm{H}_{30} \mathrm{O}_{9} \mathrm{SFe} 562.0960$. Found $585.0855[\mathrm{M}+\mathrm{Na}]^{+}$.

[2,3,6-Tri- $O$-acetyl-4- $O$-(2',3', ,', ,' '-tetra- $O$-acetyl- $\beta$-D-galactopyranosyl)- $\beta$-D-glucopyranosyl]thiomethylferrocene (7). Starting from 1 (50 mg, $0.231 \mathrm{mmol})$, column chromatography (EtherHexane 4:1) gave 7 (184 mg, 93\%) as a yellow solid: M. p. $89^{\circ} \mathrm{C} ;[\alpha]_{\mathrm{D}}-17^{\circ}\left(c 1, \mathrm{CHCl}_{3}\right)$; IR $\left(\mathrm{KBr}, \mathrm{cm}^{-1}\right)$ :

\footnotetext{
${ }^{3}$ Matta, K. L.; Girotra, R. N.; Barlow, J. J. Carbohydr. Res. 1975, 43, 101-109.

${ }^{4}$ a) Kaufman, R. J.; Sidhu, R. S. J. Org. Chem. 1982, 47, 4941-4947; b) Mereyala, H. B.; Gurrala, S. R. Carbohydr. Res. 1998, 307, 351-354.

${ }^{5}$ Chemyak, A. Y.; Sharma, G. V. M.; Kononov, L. O.; Krishna, P. R.; Levinsky, A. B.; Kochetkov, N. K.; Rao, A. V. R. Carbohydr. Res. 1992, 223, 303-309.
} 
2953, 2924, 2853, 1751, 1370, 1230, 1047; ${ }^{1} \mathrm{H}-\mathrm{NMR}\left(300 \mathrm{MHz}, \mathrm{CDCl}_{3}\right), \delta$ (ppm): 5.33 (bd, $1 \mathrm{H},{ }^{3} J=2.8$ Hz, H-4'), 5.14 (t, 1H, ${ }^{3} J=9.1 \mathrm{~Hz}, \mathrm{H}-3$ ), 5.08 (dd, $1 \mathrm{H},{ }^{3} J_{2^{2}, 3^{3}}=10.4 \mathrm{~Hz},{ }^{3} J_{1^{\prime}, 2^{\prime}}=7.8 \mathrm{~Hz}, \mathrm{H}-2^{\prime}$ ), 4.93 (dd,

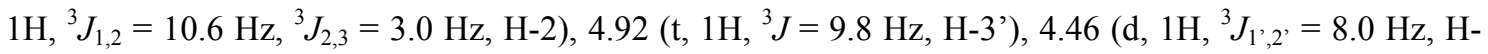
1'), 4.46 (bd, $\left.1 \mathrm{H},{ }^{2} J_{6,6}=13.5 \mathrm{~Hz}, \mathrm{H}-6\right), 4.36\left(\mathrm{~d}, 1 \mathrm{H},{ }^{3} J_{1,2}=10.5 \mathrm{~Hz}, \mathrm{H}-1\right), 4.18$ (bs, $1 \mathrm{H}, \mathrm{H}_{\mathrm{Cp}}$ ), $4.12(\mathrm{~s}, 5 \mathrm{H}$, $\mathrm{H}_{\mathrm{Cp}}$ ), 4.10-4.02 (m, 6H, $\mathrm{H}_{\mathrm{Cp}}, \mathrm{H}-6,6$ ', $6^{\prime}$ ), 3.85 (t, $1 \mathrm{H},{ }^{3} J=7.0 \mathrm{~Hz}, \mathrm{H}-5$ ') 3.78 (t, $\left.1 \mathrm{H},{ }^{3} J=9.5 \mathrm{~Hz}, \mathrm{H}-4\right)$, $3.69\left(\mathrm{~d}, 1 \mathrm{H},{ }^{2} J=13.0 \mathrm{~Hz}, \mathrm{CH}_{2} \mathrm{~S}\right), 3.60\left(\mathrm{~d}, 1 \mathrm{H},{ }^{2} J=13.2 \mathrm{~Hz}, \mathrm{CH}_{2} \mathrm{~S}\right), 3.54\left(\mathrm{ddd}, 1 \mathrm{H},{ }^{3} J_{4,5}=9.8 \mathrm{~Hz},{ }^{3} J_{5,6}=\right.$ $5.5 \mathrm{~Hz},{ }^{3} J_{5,6}=1.7 \mathrm{~Hz}, \mathrm{H}-5$ ), 2.14 (s, 3H, $\mathrm{CH}_{3} \mathrm{CO}$ ), 2.13 (s, 3H, $\mathrm{CH}_{3} \mathrm{CO}$ ), 2.04 (s, 6H, $\mathrm{CH}_{3} \mathrm{CO}$ ), 2.02 (s, $3 \mathrm{H}, \mathrm{CH}_{3} \mathrm{CO}$ ), 1.99 (s, $3 \mathrm{H}, \mathrm{CH}_{3} \mathrm{CO}$ ), 1.95 (s, 3H, $\mathrm{CH}_{3} \mathrm{CO}$ ); ${ }^{13} \mathrm{C}-\mathrm{NMR}\left(75 \mathrm{MHz}, \mathrm{CDCl}_{3}\right.$ ), $\delta(\mathrm{ppm}): 170.3$, 170.1, 170.0, 169.6, 169.5169 .0 (CO), 101.0 (C-1'), 83.4 (C $\mathrm{C}_{\text {ipso }}$ ), 82.1 (C-1), 76.5 (C-5), 76.2 (C-4), 73.7 (C-3), 70.9 (C-3'), 70.6 (C-5'), $70.2(\mathrm{C}-2), 69.0\left(\mathrm{C}^{2}{ }^{\prime}\right), 68.8\left(\mathrm{C}_{\mathrm{Cp}_{\mathrm{p}}}\right), 68.7\left(\mathrm{C}_{\mathrm{Cp}^{\prime}}\right)$, 68.5, 68.4, $67.9\left(\mathrm{C}_{\mathrm{Cp}_{\mathrm{p}}}\right)$, 66.5 (C-4'), 62.3 (C-6), 60.7 (C-6'), $29.6\left(\mathrm{CH}_{2} \mathrm{~S}\right), 20.8,20.7,20.6,20.5,20.4\left(\mathrm{CH}_{3} \mathrm{CO}\right)$; HMRS (FAB+): Calc. for $\mathrm{C}_{37} \mathrm{H}_{46} \mathrm{O}_{17} \mathrm{SFe} 850.1805$. Found $873.1701[\mathrm{M}+\mathrm{Na}]^{+}$.

1,1'-Bis[(2,3,4,6-tetra- $O$-acetyl- $\beta$-D-glucopyranosyl)thiomethyl]ferrocene (9). Starting from 8 (50 mg, $0.203 \mathrm{mmol}$ ), column chromatography (EtOAc-Hexane 1:2 $\rightarrow 3: 2)$ gave $\mathbf{9}(175 \mathrm{mg}, 92 \%)$ as a yellow solid: M. p. $81{ }^{\circ} \mathrm{C}$; $[\alpha]_{\mathrm{D}}-37^{\circ}\left(c 1, \mathrm{CHCl}_{3}\right)$; IR $\left(\mathrm{KBr}, \mathrm{cm}^{-1}\right): 2945,2868,1751,1370,1225,1037$; ${ }^{1} \mathrm{H}-\mathrm{NMR}\left(300 \mathrm{MHz}, \mathrm{CDCl}_{3}\right.$ ), $\delta$ (ppm): 5.19 (t, 2H, $\left.{ }^{3} \mathrm{~J}=9.2 \mathrm{~Hz}, \mathrm{H}-3\right), 5.09$ (t, $\left.2 \mathrm{H},{ }^{3} J=9.5 \mathrm{~Hz}, \mathrm{H}-4\right), 5.04$ (t, $\left.2 \mathrm{H},{ }^{3} J=9.5 \mathrm{~Hz}, \mathrm{H}-2\right), 4.41\left(\mathrm{~d}, 2 \mathrm{H},{ }^{3} J_{1,2}=9.9 \mathrm{~Hz}, \mathrm{H}-1\right), 4.26\left(\mathrm{dd}, 2 \mathrm{H},{ }^{2} J_{6,6}=12.4 \mathrm{~Hz},{ }^{3} J_{5,6}=4.8 \mathrm{~Hz}, \mathrm{H}-\right.$ 6), 4.17 (m, 2H, H-6'), 4.13 (bs, 8H, $\mathrm{H}_{\mathrm{Cp}}$ ), 3.71 (d, $2 \mathrm{H},{ }^{2} J=13.0 \mathrm{~Hz}, \mathrm{CH}_{2} \mathrm{~S}$ ), 3.69-3.63 (m, 2H, H-5), 3.65 (d, $2 \mathrm{H},{ }^{2} J=13.1 \mathrm{~Hz}, \mathrm{CH}_{2} \mathrm{~S}$ ), 2.12 (s, 6H, $\mathrm{CH}_{3} \mathrm{CO}$ ), 2.03 (s, 6H, $\mathrm{CH}_{3} \mathrm{CO}$ ), 2.02 (s, 6H, $\mathrm{CH}_{3} \mathrm{CO}$ ), 2.00 (s, $\left.6 \mathrm{H}, \mathrm{CH}_{3} \mathrm{CO}\right) ;{ }^{13} \mathrm{C}-\mathrm{NMR}\left(75 \mathrm{MHz}, \mathrm{CDCl}_{3}\right), \delta(\mathrm{ppm}): 170.6,170.1,169.4(\mathrm{CO}), 84.0\left(\mathrm{C}_{\mathrm{ipso}}\right), 82.5(\mathrm{C}-1)$, 75.8 (C-5), 73.8 (C-3), 69.7 (C-2), 69.6, 69.4, 69.3, $68.9\left(\mathrm{C}_{\mathrm{Cp}}\right), 68.4(\mathrm{C}-4), 62.3(\mathrm{C}-6), 29.2\left(\mathrm{CH}_{2} \mathrm{~S}\right), 20.8$, 20.7, $20.6\left(\mathrm{CH}_{3} \mathrm{CO}\right)$; HMRS (FAB+): Calc. for $\mathrm{C}_{40} \mathrm{H}_{50} \mathrm{O}_{18} \mathrm{~S}_{2} \mathrm{Fe}$ 938.1788. Found $961.1686[\mathrm{M}+\mathrm{Na}]^{+}$.

1,1'-Bis[(2,3,4,6-tetra- $O$-acetyl- $\alpha$-D-mannopyranosyl)thiomethyl]ferrocene (10). Starting from 8 (50 mg, $0.203 \mathrm{mmol}$ ), column chromatography (EtOAc-Hexane 1:2 $\rightarrow$ 3:2) gave 10 (167 mg, $87 \%$ ) as a yellow solid: M. p. $147^{\circ} \mathrm{C} ;[\alpha]_{\mathrm{D}}+79^{\circ}\left(c 1, \mathrm{CHCl}_{3}\right)$; IR $\left(\mathrm{KBr}, \mathrm{cm}^{-1}\right): 2950,2925,1749,1369$, 1226, 1049; ${ }^{1} \mathrm{H}-\mathrm{NMR}\left(300 \mathrm{MHz}, \mathrm{CDCl}_{3}\right.$ ), $\delta$ (ppm): 5.34-5.29 (m, 2H, H-4), 5.28 (bs, 2H, H-2), 5.23 (dd, $\left.2 \mathrm{H},{ }^{3} J_{3,4}=10.0 \mathrm{~Hz},{ }^{3} J_{2,3}=2.3 \mathrm{~Hz}, \mathrm{H}-3\right), 5.19$ (bs, $\left.2 \mathrm{H}, \mathrm{H}-1\right), 4.36-4.31$ (m, $\left.2 \mathrm{H}, \mathrm{H}-5\right), 4.31$ (dd, $2 \mathrm{H},{ }^{2} J_{6,6}{ }=$ $\left.10.9 \mathrm{~Hz},{ }^{3} J_{5,6}=4.8 \mathrm{~Hz}, \mathrm{H}-6\right), 4.18$ (bs, $\left.2 \mathrm{H}, \mathrm{H}_{\mathrm{Cp}}\right), 4.16$ (d, $2 \mathrm{H},{ }^{3} J=1.5 \mathrm{~Hz}, \mathrm{H}_{\mathrm{Cp}}$ ), $4.12\left(\mathrm{t}, 4 \mathrm{H},{ }^{3} J=1.7 \mathrm{~Hz}\right.$, $\left.\mathrm{H}_{\mathrm{Cp}}\right), 4.07\left(\mathrm{~d}, 2 \mathrm{H},{ }^{2} J=10.1 \mathrm{~Hz}, \mathrm{H}-6\right.$ ) $), 3.62\left(\mathrm{~d}, 2 \mathrm{H},{ }^{2} J=13.6 \mathrm{~Hz}, \mathrm{CH}_{2} \mathrm{~S}\right), 3.55\left(\mathrm{~d}, 2 \mathrm{H},{ }^{2} J=13.6 \mathrm{~Hz}\right.$, $\mathrm{CH}_{2} \mathrm{~S}$ ), 2.14 (s, 6H, $\mathrm{CH}_{3} \mathrm{CO}$ ), 2.13 (s, 6H, $\mathrm{CH}_{3} \mathrm{CO}$ ), 2.04 (s, 6H, $\mathrm{CH}_{3} \mathrm{CO}$ ), 1.97 (s, 6H, $\mathrm{CH}_{3} \mathrm{CO}$ ); ${ }^{13} \mathrm{C}-$ NMR (75 MHz, $\left.\mathrm{CDCl}_{3}\right), \delta(\mathrm{ppm}): 170.5,169.8,169.7,169.6(\mathrm{CO}), 84.0\left(\mathrm{C}_{\mathrm{ipso}}\right), 81.9(\mathrm{C}-1), 70.7(\mathrm{C}-2)$, $69.6(\mathrm{C}-3), 69.5\left(\mathrm{C}-5, \mathrm{C}_{\mathrm{Cp}}\right), 69.4,69.1,69.0\left(\mathrm{C}_{\mathrm{Cp}}\right), 66.3(\mathrm{C}-4), 62.4(\mathrm{C}-6), 30.6\left(\mathrm{CH}_{2} \mathrm{~S}\right), 20.9,20.8,20.7$, $20.6\left(\mathrm{CH}_{3} \mathrm{CO}\right)$; MALDI-TOF: Calc. for $\mathrm{C}_{40} \mathrm{H}_{50} \mathrm{O}_{18} \mathrm{~S}_{2} \mathrm{Fe}$ 938.179. Found $938.174[\mathrm{M}]^{+}, 939.168[\mathrm{M}+\mathrm{H}]^{+}$, $961.182[\mathrm{M}+\mathrm{Na}]^{+}$.

\section{1,1'-Bis $\{[2,3,6$-tri- $O$-acetyl-4- $O$-(2',3',4',6'-tetra- $O$-acetyl- $\beta$-D-galactopyranosyl)- $\beta$-D-gluco-} pyranosyl]thiomethyl\}ferrocene (11). Starting from 8 (30 $\mathrm{mg}, 0.122 \mathrm{mmol})$, column chromatography (EtOAc-Hexane 2:1) gave 11 (168 mg, 91\%) as a yellow solid: M. p. $112{ }^{\circ} \mathrm{C} ;[\alpha]_{\mathrm{D}}-21^{\circ}\left(c 1, \mathrm{CHCl}_{3}\right)$; IR 
$\left(\mathrm{KBr}, \mathrm{cm}^{-1}\right): 2938,2871,1735,1370,1232,1046 ;{ }^{1} \mathrm{H}-\mathrm{NMR}\left(300 \mathrm{MHz}, \mathrm{CDCl}_{3}\right), \delta(\mathrm{ppm}): 5.25$ (bs, 2H, H$\left.4^{\prime}\right), 5.08\left(\mathrm{t}, 2 \mathrm{H},{ }^{3} \mathrm{~J}=9.2 \mathrm{~Hz}, \mathrm{H}-3\right), 5.01\left(\mathrm{dd}, 2 \mathrm{H},{ }^{3} \mathrm{~J}_{2^{\prime}, 3^{\prime}}=10.3 \mathrm{~Hz},{ }^{3} J_{1^{\prime}, 2^{\prime}}=7.9 \mathrm{~Hz}, \mathrm{H}-2^{\prime}\right), 4.88\left(\mathrm{dd}, 2 \mathrm{H},{ }^{3} J_{1,2}\right.$ $\left.=10.3 \mathrm{~Hz},{ }^{3} J_{2,3}=3.4 \mathrm{~Hz}, \mathrm{H}-2\right), 4.84$ (t, $\left.2 \mathrm{H},{ }^{3} J=9.7 \mathrm{~Hz}, \mathrm{H}-3^{\prime}\right), 4.42$ (d, $\left.2 \mathrm{H},{ }^{3} J_{1^{\prime}, 2^{\prime}}=7.6 \mathrm{~Hz}, \mathrm{H}-1^{\prime}\right), 4.39$ (bd, $\left.2 \mathrm{H},{ }^{2} J_{6,6}=9.5 \mathrm{~Hz}, \mathrm{H}-6\right), 4.31\left(\mathrm{~d}, 2 \mathrm{H},{ }^{3} J_{1,2}=10.0 \mathrm{~Hz}, \mathrm{H}-1\right), 4.08$ (bs, $2 \mathrm{H}, \mathrm{H}_{\mathrm{Cp}}$ ), 4.03 (bs, $12 \mathrm{H}, \mathrm{H}_{\mathrm{Cp}}, \mathrm{H}-$ 6, 6', 6'), 3.81 (t, $2 \mathrm{H},{ }^{3} J=6.7 \mathrm{~Hz}, \mathrm{H}-5$ ') 3.71 (t, $\left.2 \mathrm{H},{ }^{3} \mathrm{~J}=9.5 \mathrm{~Hz}, \mathrm{H}-4\right), 3.60$ (d, $2 \mathrm{H},{ }^{2} J=13.1 \mathrm{~Hz}, \mathrm{CH}_{2} \mathrm{~S}$ ), $3.52\left(\mathrm{~d}, 2 \mathrm{H},{ }^{2} \mathrm{~J}=12.9 \mathrm{~Hz}, \mathrm{CH}_{2} \mathrm{~S}\right.$ ), 3.52-3.47 (m, 2H, H-5), 2.07 (s, 6H, $\mathrm{CH}_{3} \mathrm{CO}$ ), 2.06 (s, 6H, $\mathrm{CH}_{3} \mathrm{CO}$ ), 1.97 (s, $12 \mathrm{H}, \mathrm{CH}_{3} \mathrm{CO}$ ), 1.95 (s, 6H, $\left.\mathrm{CH}_{3} \mathrm{CO}\right), 1.93$ (s, 6H, $\mathrm{CH}_{3} \mathrm{CO}$ ), 1.88 (s, $6 \mathrm{H}, \mathrm{CH}_{3} \mathrm{CO}$ ); ${ }^{13} \mathrm{C}-\mathrm{NMR}(75$ $\left.\mathrm{MHz}, \mathrm{CDCl}_{3}\right), \delta(\mathrm{ppm}): 170.1,169.9,169.8,169.4,169.3,168.8(\mathrm{CO}), 100.8\left(\mathrm{C}-1^{\prime}\right), 83.8\left(\mathrm{C}_{\mathrm{ipso}}\right), 82.0(\mathrm{C}-$ 1), 76.4 (C-5), 76.0 (C-4), 73.5 (C-3), 70.7 (C-3'), 70.4 (C-5'), 69.9 (C-2), 69.3 (C-2'), 69.1, 68.9, 68.6 $\left(\mathrm{C}_{\mathrm{Cp}}\right), 66.4$ (C-4'), 62.1 (C-6), 60.6 (C-6'), $29.1\left(\mathrm{CH}_{2} \mathrm{~S}\right), 20.7,20.5,20.4,20.3,20.2\left(\mathrm{CH}_{3} \mathrm{CO}\right)$; HMRS $(\mathrm{FAB}+)$ : Calc. for $\mathrm{C}_{64} \mathrm{H}_{82} \mathrm{O}_{34} \mathrm{~S}_{2} \mathrm{Fe} 1514.3478$. Found $1537.3379[\mathrm{M}+\mathrm{Na}]^{+}$.

\section{General procedure for the Zemplén de- $O$-acetylation of thioglycosylated ferrocenes 5-7 and}

9-11. A solution of thioglycosylated ferrocene 5-7 or 9-11 in dry $\mathrm{MeOH}(15-20 \mathrm{~mL})$ or in a mixture of dry $\mathrm{MeOH}$ and dry $\mathrm{CH}_{2} \mathrm{Cl}_{2}$ 10:1 (15-20 mL) was made alkaline to $\mathrm{pH}$ 9-10 (indicator paper) with a fresh methanolic solution of $\mathrm{NaOMe}(1 \mathrm{M})$ and stirred at room temperature for 2-24 $\mathrm{h}$ following the reaction by TLC. If the product precipitated, the solvent was concentrated under vacuum to $1-2 \mathrm{~mL}$ and ether was added. The solid was filtered off and washed with ether. In other case, the solvent was removed under vacuum and the crude was purified by column chromatography. The pure compound was dissolved in water and lyophilised.

$\beta$-D-Glucopyranosylthiomethylferrocene (12). Starting from 5 (173 $\mathrm{mg}, 0.308 \mathrm{mmol})$ in dry $\mathrm{MeOH}$, column chromatography (EtOAc-MeOH 3:2) gave 12 (118 mg, 98\%) as a yellow solid: M. p. 145 ${ }^{\circ} \mathrm{C}$; $[\alpha]_{\mathrm{D}}-40^{\circ}$ (c 0.25, MeOH); IR (KBr, cm $\left.{ }^{-1}\right): 3369,2922,2856,1019 ;{ }^{1} \mathrm{H}-\mathrm{NMR}\left(300 \mathrm{MHz}, \mathrm{D}_{2} \mathrm{O}, 60\right.$ $\left.{ }^{\circ} \mathrm{C}\right), \delta(\mathrm{ppm}): 4.79\left(\mathrm{~d}, 1 \mathrm{H},{ }^{3} J_{1,2}=9.7 \mathrm{~Hz}, \mathrm{H}-1\right), 4.71\left(\mathrm{bs}, 2 \mathrm{H}, \mathrm{H}_{\mathrm{Cp}}\right), 4.68\left(\mathrm{bs}, 2 \mathrm{H}, \mathrm{H}_{\mathrm{Cp}}\right), 4.63\left(\mathrm{~s}, 5 \mathrm{H}, \mathrm{H}_{\mathrm{Cp}}\right)$, $4.24\left(\mathrm{~d}, 1 \mathrm{H},{ }^{2} J_{6,6^{\prime}}=12.5 \mathrm{~Hz}, \mathrm{H}-6\right), 4.21\left(\mathrm{~d}, 1 \mathrm{H},{ }^{2} J=13.3 \mathrm{~Hz}, \mathrm{CH}_{2} \mathrm{~S}\right), 4.15\left(\mathrm{~d}, 1 \mathrm{H},{ }^{2} J=13.3 \mathrm{~Hz}, \mathrm{CH}_{2} \mathrm{~S}\right)$, $4.06\left(\mathrm{dd}, 1 \mathrm{H},{ }^{2} J_{6,6^{\prime}}=12.5 \mathrm{~Hz},{ }^{3} J_{5,6^{\prime}}=4.9 \mathrm{~Hz}, \mathrm{H}-6^{\prime}\right), 3.84-3.75(\mathrm{~m}, 3 \mathrm{H}, \mathrm{H}-3,4,5), 3.67$ (t, $1 \mathrm{H},{ }^{3} \mathrm{~J}=9.4 \mathrm{~Hz}$, $\mathrm{H}-2) ;{ }^{13} \mathrm{C}-\mathrm{NMR}\left(75 \mathrm{MHz}, \mathrm{D}_{2} \mathrm{O}, 60{ }^{\circ} \mathrm{C}\right), \delta(\mathrm{ppm}): 85.0(\mathrm{C}-1), 84.9\left(\mathrm{C}_{\mathrm{ipso}}\right), 80.2(\mathrm{C}-5), 77.8(\mathrm{C}-3), 72.7$ (C2), $70.1(\mathrm{C}-4), 69.5\left(\mathrm{C}_{\mathrm{Cp}}\right), 69.3\left(\mathrm{C}_{\mathrm{Cp}}\right), 69.1,68.8,68.5\left(\mathrm{C}_{\mathrm{Cp}}\right), 61.4(\mathrm{C}-6), 29.9\left(\mathrm{CH}_{2} \mathrm{~S}\right)$; HMRS $(\mathrm{FAB}+)$ : Calc. for $\mathrm{C}_{17} \mathrm{H}_{22} \mathrm{O}_{5} \mathrm{SFe}$ 394.0538. Found $417.0433[\mathrm{M}+\mathrm{Na}]^{+}$.

Q-D-Mannopyranosylthiomethylferrocene (13). Starting from 6 (104 mg, $0.185 \mathrm{mmol}$ ) in dry $\mathrm{MeOH}$, column chromatography (EtOAc-MeOH 3:2) gave 13 (69 mg, 95\%) as a yellow solid: M. p. 168 ${ }^{\circ} \mathrm{C}$; $[\alpha]_{\mathrm{D}}+154^{\circ}$ (c 0.25, MeOH); IR (KBr, cm $\left.{ }^{-1}\right): 3396,2922,2858,1069 ;{ }^{1} \mathrm{H}-\mathrm{NMR}\left(300 \mathrm{MHz}, \mathrm{CD}_{3} \mathrm{OD}\right)$, $\delta$ (ppm): $5.18\left(\mathrm{~d}, 1 \mathrm{H},{ }^{3} J_{1,2}=1.3 \mathrm{~Hz}, \mathrm{H}-1\right), 4.25\left(\mathrm{dd}, 1 \mathrm{H},{ }^{3} J=3.5 \mathrm{~Hz},{ }^{3} J=1.5 \mathrm{~Hz}, \mathrm{H}_{\mathrm{Cp}}\right), 4.22\left(\mathrm{dd}, 1 \mathrm{H},{ }^{3} J=\right.$ $3.5 \mathrm{~Hz},{ }^{3} J=1.6 \mathrm{~Hz}, \mathrm{H}_{\mathrm{Cp}}$ ), $4.15\left(\mathrm{~s}, 5 \mathrm{H}, \mathrm{H}_{\mathrm{Cp}}\right.$ ), $4.12\left(\mathrm{t}, 2 \mathrm{H},{ }^{3} J=1.6 \mathrm{~Hz}, \mathrm{H}_{\mathrm{Cp}}\right), 3.94-3.90$ (m, 1H, H-5), 3.86 $\left(\mathrm{dd}, 1 \mathrm{H},{ }^{2} J_{6,6}=11.8 \mathrm{~Hz},{ }^{3} J_{5,6}=2.3 \mathrm{~Hz}, \mathrm{H}-6\right), 3.82\left(\mathrm{dd}, 1 \mathrm{H},{ }^{3} J_{2,3}=3.0 \mathrm{~Hz},{ }^{3} J_{1,2}=1.5 \mathrm{~Hz}, \mathrm{H}-2\right), 3.75$ (dd, $1 \mathrm{H},{ }^{2} J_{6,6^{\prime}}=11.8 \mathrm{~Hz},{ }^{3} J_{5,6^{\prime}}=5.9 \mathrm{~Hz}, \mathrm{H}-6$ '), 3.69 (d, $\left.1 \mathrm{H},{ }^{2} J=13.6 \mathrm{~Hz}, \mathrm{CH}_{2} \mathrm{~S}\right), 3.68-3.62$ (m, 2H, H-3, 4), $3.59\left(\mathrm{~d}, 1 \mathrm{H},{ }^{2} J=13.6 \mathrm{~Hz}, \mathrm{CH}_{2} \mathrm{~S}\right) ;{ }^{13} \mathrm{C}-\mathrm{NMR}\left(75 \mathrm{MHz}, \mathrm{CD}_{3} \mathrm{OD}\right), \delta(\mathrm{ppm}): 85.9\left(\mathrm{C}_{\mathrm{ipso}}\right), 85.4(\mathrm{C}-1), 74.9$ 
(C-5), $73.5(\mathrm{C}-2), 73.3(\mathrm{C}-3), 70.2\left(\mathrm{C}_{\mathrm{Cp}}\right), 69.7\left(\mathrm{C}_{\mathrm{Cp}}\right), 69.6\left(\mathrm{C}_{\mathrm{Cp}}\right), 69.4(\mathrm{C}-4), 68.9,68.8\left(\mathrm{C}_{\mathrm{Cp}}\right), 62.8(\mathrm{C}-6)$, $31.0\left(\mathrm{CH}_{2} \mathrm{~S}\right)$; HMRS $(\mathrm{FAB}+)$ : Calc. for $\mathrm{C}_{17} \mathrm{H}_{22} \mathrm{O}_{5} \mathrm{SFe}$ 394.0538. Found $417.0433[\mathrm{M}+\mathrm{Na}]^{+}$.

4-O-( $\beta$-D-Galactopyranosyl)- $\beta$-D-glucopyranosylthiomethylferrocene (14). Starting from 7 (158 mg, $0.186 \mathrm{mmol})$ in dry MeOH, column chromatography (EtOAc-MeOH 2:1) gave 14 (78 mg, 76\%) as a yellow solid: M. p. $166^{\circ} \mathrm{C}$ (decomposition); $[\alpha]_{\mathrm{D}}-28^{\circ}\left(c\right.$ 0.25, MeOH); $\mathrm{IR}\left(\mathrm{KBr}, \mathrm{cm}^{-1}\right): 3402,2918$, 2859, 1077; ${ }^{1} \mathrm{H}-\mathrm{NMR}\left(300 \mathrm{MHz}, \mathrm{CD}_{3} \mathrm{OD}\right), \delta(\mathrm{ppm}): 4.36$ (d, $1 \mathrm{H},{ }^{3} J_{1^{\prime}, 2^{\prime}}=7.3 \mathrm{~Hz}, \mathrm{H}-1$ '), 4.34 (d, $1 \mathrm{H},{ }^{3} J_{1,2}$ $=9.7 \mathrm{~Hz}, \mathrm{H}-1), 4.25\left(\mathrm{dd}, 1 \mathrm{H},{ }^{3} \mathrm{~J}=3.4 \mathrm{~Hz},{ }^{3} J=1.5 \mathrm{~Hz}, \mathrm{H}_{\mathrm{Cp}}\right), 4.22\left(\mathrm{dd}, 1 \mathrm{H},{ }^{3} \mathrm{~J}=3.4 \mathrm{~Hz},{ }^{3} J=1.5 \mathrm{~Hz}, \mathrm{H}_{\mathrm{Cp}}\right)$, $4.15\left(\mathrm{~s}, 5 \mathrm{H}, \mathrm{H}_{\mathrm{Cp}}\right), 4.12\left(\mathrm{t}, 2 \mathrm{H},{ }^{3} J=1.8 \mathrm{~Hz}, \mathrm{H}_{\mathrm{Cp}}\right), 3.94\left(\mathrm{dd}, 1 \mathrm{H},{ }^{3} J_{6,6}=12.2 \mathrm{~Hz},{ }^{3} J_{5,6}=2.4 \mathrm{~Hz}, \mathrm{H}-6\right), 3.83$ (dd, $\left.1 \mathrm{H},{ }^{2} J_{6,6}=12.0 \mathrm{~Hz},{ }^{3} J_{5,6}=4.3 \mathrm{~Hz}, \mathrm{H}-6\right), 3.82\left(\mathrm{~d}, 1 \mathrm{H},{ }^{2} J=13.2 \mathrm{~Hz}, \mathrm{CH}_{2} \mathrm{~S}\right.$ ), 3.81 (bs, $1 \mathrm{H}, \mathrm{H}-4$ '), 3.78 (dd, $\left.1 \mathrm{H},{ }^{2} J_{6^{\prime}, 6^{\prime}}=11.3 \mathrm{~Hz},{ }^{3} J_{5^{\prime}, 6^{\prime}}=7.4 \mathrm{~Hz}, \mathrm{H}-6^{\prime}\right), 3.70\left(\mathrm{dd}, 1 \mathrm{H},{ }^{2} J_{6^{\prime}, 6^{\prime}}=11.3 \mathrm{~Hz},{ }^{3} J_{5^{\prime}, 6^{\prime}}=4.4 \mathrm{~Hz}, \mathrm{H}-6^{\prime}\right), 3.69$ (d, $1 \mathrm{H},{ }^{2} J=13.2 \mathrm{~Hz}, \mathrm{CH}_{2} \mathrm{~S}$ ), 3.60-3.57 (m, $1 \mathrm{H}, \mathrm{H}-5$ ) $), 3.58$ (t, $\left.1 \mathrm{H},{ }^{3} J=9.2 \mathrm{~Hz}, \mathrm{H}-4\right), 3.55$ (dd, $1 \mathrm{H},{ }^{3} J_{2},{ }^{\prime},=$ $\left.9.6 \mathrm{~Hz},{ }^{3} J_{1^{\prime}, 2^{\prime}}=7.4 \mathrm{~Hz}, \mathrm{H}-2^{\prime}\right), 3.48\left(\mathrm{dd}, 1 \mathrm{H},{ }^{3} J_{2^{\prime}, 3^{\prime}}=9.7 \mathrm{~Hz},{ }^{3} J_{3^{\prime}, 4^{\prime}}=3.3 \mathrm{~Hz}, \mathrm{H}-3^{\prime}\right), 3.46$ (t, $1 \mathrm{H},{ }^{3} J=8.7 \mathrm{~Hz}$, H-3), 3.37 (ddd, $1 \mathrm{H},{ }^{3} J_{4,5}=9.6 \mathrm{~Hz},{ }^{3} J_{5,6}=4.3 \mathrm{~Hz},{ }^{3} J_{5,6}=2.5 \mathrm{~Hz}, \mathrm{H}-5$ ), 3.28 (dd, $1 \mathrm{H},{ }^{3} J_{1,2}=9.7 \mathrm{~Hz},{ }^{3} J_{2,3}=$ $8.8 \mathrm{~Hz}, \mathrm{H}-2) ;{ }^{13} \mathrm{C}-\mathrm{NMR}\left(75 \mathrm{MHz}, \mathrm{CD}_{3} \mathrm{OD}\right), \delta(\mathrm{ppm}): 105.0$ (C-1'), 85.8 (C-1), 85.7 (C $\mathrm{C}_{\mathrm{ipso}}$ ), 80.5 (C-4), 80.4 (C-5), 78.0 (C-3), 77.1 (C-5'), 74.8 (C-3’), 74.1 (C-2), 72.5 (C-2'), 70.3 (C-4'), 70.2, 69.8 (C ( $\mathrm{Cp}_{\mathrm{Cp}}$ ), $69.7\left(\mathrm{C}_{\mathrm{Cp}^{\mathrm{p}}}\right), 69.1,68.8\left(\mathrm{C}_{\mathrm{Cp}}\right), 62.5\left(\mathrm{C}^{-6}{ }^{\prime}\right), 62.1(\mathrm{C}-6), 30.4\left(\mathrm{CH}_{2} \mathrm{~S}\right)$; HMRS (FAB+): Calc. for $\mathrm{C}_{23} \mathrm{H}_{32} \mathrm{O}_{10} \mathrm{SFe}$ 556.1066. Found $579.0963[\mathrm{M}+\mathrm{Na}]^{+}$.

1,1'-Bis( $\beta$-D-glucopyranosylthiomethyl)ferrocene (15). Starting from 9 (176 mg, $0.187 \mathrm{mmol}$ ) in dry $\mathrm{MeOH}$, column chromatography (EtOAc-MeOH 2:1) gave 15 (108 mg, 96\%) as a yellow solid: M. p. $65^{\circ} \mathrm{C}$ (decomposition); $[\alpha]_{\mathrm{D}}-32^{\circ}(c 0.25, \mathrm{MeOH})$; IR $\left(\mathrm{KBr}, \mathrm{cm}^{-1}\right): 3396,2920,2880,1032 ;{ }^{1} \mathrm{H}-\mathrm{NMR}$ $\left(300 \mathrm{MHz}, \mathrm{D}_{2} \mathrm{O}, 60^{\circ} \mathrm{C}\right), \delta(\mathrm{ppm}): 4.78\left(\mathrm{~d}, 2 \mathrm{H},{ }^{3} J_{1,2}=9.7 \mathrm{~Hz}, \mathrm{H}-1\right), 4.70\left(\mathrm{~m}, \mathrm{H}_{\mathrm{Cp}}, \mathrm{HDO}\right), 4.64\left(\mathrm{~d}, 4 \mathrm{H},{ }^{3} J=\right.$ $\left.7.7 \mathrm{~Hz}, \mathrm{H}_{\mathrm{Cp}}\right), 4.24\left(\mathrm{~d}, 2 \mathrm{H},{ }^{2} J_{6,6}=12.5 \mathrm{~Hz}, \mathrm{H}-6\right), 4.20$ (bdd, $\left.4 \mathrm{H},{ }^{2} J=11.8 \mathrm{~Hz}, \mathrm{CH}_{2} \mathrm{~S}\right), 4.06\left(\mathrm{dd}, 2 \mathrm{H},{ }^{2} J_{6,6}{ }=\right.$ $\left.12.9 \mathrm{~Hz},{ }^{3} J_{5,6}=4.5 \mathrm{~Hz}, \mathrm{H}-6^{\prime}\right), 3.84-3.75(\mathrm{~m}, 6 \mathrm{H}, \mathrm{H}-3,4,5), 3.67$ (t, $\left.2 \mathrm{H},{ }^{3} \mathrm{~J}=9.3 \mathrm{~Hz}, \mathrm{H}-2\right) ;{ }^{13} \mathrm{C}-\mathrm{NMR}(75$ $\left.\mathrm{MHz}, \mathrm{D}_{2} \mathrm{O}, 60^{\circ} \mathrm{C}\right), \delta(\mathrm{ppm}): 85.4\left(\mathrm{C}_{\mathrm{ipso}}\right), 85.0(\mathrm{C}-1), 80.2$ (C-5), 77.8 (C-3), 72.7 (C-2), 70.3, 70.1, 69.9, 69.7, $69.5\left(\mathrm{C}_{\mathrm{Cp}}, \mathrm{C}-4\right), 61.5(\mathrm{C}-6), 29.7\left(\mathrm{CH}_{2} \mathrm{~S}\right)$; HMRS (FAB+): Calc. for $\mathrm{C}_{24} \mathrm{H}_{34} \mathrm{O}_{10} \mathrm{~S}_{2} \mathrm{Fe}$ 602.0943. Found $625.0830[\mathrm{M}+\mathrm{Na}]^{+}$.

1,1'-Bis( $\alpha$-D-mannopyranosylthiomethyl)ferrocene (16). Starting from 10 (162 mg, 0.173 mmol) in dry $\mathrm{MeOH}-\mathrm{CH}_{2} \mathrm{Cl}_{2}$ 10:1, column chromatography (EtOAc-MeOH 2:1) gave 16 (99 mg, 95\%) as a yellow solid: M. p. $74{ }^{\circ} \mathrm{C} ;[\alpha]_{\mathrm{D}}+190^{\circ}(c \mathrm{0.25}, \mathrm{MeOH})$; IR $\left(\mathrm{KBr}, \mathrm{cm}^{-1}\right): 3426,2919,2876,1067 ;{ }^{1} \mathrm{H}-$ NMR (300 MHz, CD 3 OD), $\delta$ (ppm): 5.18 (s, 2H, H-1), 4.22 (s, 2H, $\mathrm{H}_{\mathrm{Cp}}$ ), 4.19 (s, 2H, $\mathrm{H}_{\mathrm{Cp}}$ ), 4.13 (bs, 4H, $\mathrm{H}_{\mathrm{Cp}}$ ), 3.94-3.90 (m, 2H, H-5), 3.86 (dd, $\left.2 \mathrm{H},{ }^{2} J_{6,6}=12.1 \mathrm{~Hz},{ }^{3} J_{5,6}=2.3 \mathrm{~Hz}, \mathrm{H}-6\right), 3.83$ (bs, 2H, H-2), 3.75 $\left(\mathrm{dd}, 2 \mathrm{H},{ }^{2} J_{6,6}=11.7 \mathrm{~Hz},{ }^{3} J_{5,6}=5.8 \mathrm{~Hz}, \mathrm{H}-6^{\prime}\right), 3.68\left(\mathrm{~d}, 2 \mathrm{H},{ }^{2} J=13.6 \mathrm{~Hz}, \mathrm{CH}_{2} \mathrm{~S}\right), 3.64$ (bs, 4H, H-3, 4), $3.59\left(\mathrm{~d}, 2 \mathrm{H},{ }^{2} J=13.6 \mathrm{~Hz}, \mathrm{CH}_{2} \mathrm{~S}\right) ;{ }^{13} \mathrm{C}-\mathrm{NMR}\left(75 \mathrm{MHz}, \mathrm{CD}_{3} \mathrm{OD}\right), \delta(\mathrm{ppm}): 86.3\left(\mathrm{C}_{\mathrm{ipso}}\right), 85.4(\mathrm{C}-1), 74.9$ (C-5), 73.4 (C-2), 73.3 (C-3), 71.0, 70.5, 70.0, $69.7\left(\mathrm{C}_{\mathrm{Cp}}\right), 68.9$ (C-4), $62.8(\mathrm{C}-6), 30.8\left(\mathrm{CH}_{2} \mathrm{~S}\right)$; HMRS (FAB+): Calc. for $\mathrm{C}_{24} \mathrm{H}_{34} \mathrm{O}_{10} \mathrm{~S}_{2} \mathrm{Fe} 602.0943$. Found $625.0861[\mathrm{M}+\mathrm{Na}]^{+}$. 
1,1'-Bis[4-O-( $\beta$-D-galactopyranosyl)- $\beta$-D-glucopyranosylthiomethyl]ferrocene (17). Starting from $11(168 \mathrm{mg}, 0.111 \mathrm{mmol})$ in dry $\mathrm{MeOH}$, precipitation with ether gave $17(86 \mathrm{mg}, 83 \%)$ as a yellow solid: M. p. $179{ }^{\circ} \mathrm{C}$ (decomposition); $[\alpha]_{\mathrm{D}}-56^{\circ}$ (c 0.125, MeOH); IR $\left(\mathrm{KBr}, \mathrm{cm}^{-1}\right)$ : 3396, 2895, 1084, 1036; ${ }^{1} \mathrm{H}-\mathrm{NMR}\left(300 \mathrm{MHz}, \mathrm{DMSO}-\mathrm{d}_{6}\right.$ ), $\delta(\mathrm{ppm}): 5.24$ (d, $\left.2 \mathrm{H},{ }^{3} J=5.7 \mathrm{~Hz}, \mathrm{OH}\right), 5.10$ (bs, 2H, OH), 4.80 (bs, 2H, OH), 4.73 (s, 2H, OH), 4.69-4.64 (m, 4H, OH), 4.52 (d, $\left.2 \mathrm{H},{ }^{3} J=4.2 \mathrm{~Hz}, \mathrm{OH}\right), 4.19$ (d, $2 \mathrm{H},{ }^{3} J_{1^{\prime},{ }^{2}}$ ' $\left.=8.8 \mathrm{~Hz}, \mathrm{H}-1^{\prime}\right), 4.18\left(\mathrm{~d}, 2 \mathrm{H},{ }^{3} J_{1,2}=9.5 \mathrm{~Hz}, \mathrm{H}-1\right), 4.17\left(\mathrm{t}, 4 \mathrm{H},{ }^{3} J=1.8 \mathrm{~Hz}, \mathrm{H}_{\mathrm{Cp}}\right), 4.10\left(\mathrm{dd}, 4 \mathrm{H},{ }^{3} J=3.6 \mathrm{~Hz}\right.$, $\left.{ }^{3} J=1.8 \mathrm{~Hz}, \mathrm{H}_{\mathrm{Cp}}\right), 3.82\left(\mathrm{dd}, 2 \mathrm{H},{ }^{2} J_{6,6}=11.0 \mathrm{~Hz},{ }^{3} J_{5,6}=4.7 \mathrm{~Hz}, \mathrm{H}-6\right), 3.75\left(\mathrm{~d}, 2 \mathrm{H},{ }^{2} J=13.3 \mathrm{~Hz}, \mathrm{CH}_{2} \mathrm{~S}\right)$, 3.61-3.58 (bm, 4H, H-3', 6), 3.57 (d, 2H, ${ }^{2} J=13.3 \mathrm{~Hz}, \mathrm{CH}_{2} \mathrm{~S}$ ), 3.53-3.43 (m, 6H, H-5', 6', 6'), 3.31 (bs, 4H, H-2', 4'), 3.26 (bd, 6H, H-3, 4, 5), 3.11-3.03 (bm, 2H, H-2); ${ }^{13} \mathrm{C}-\mathrm{NMR}$ (75 MHz, DMSO-d 6 ), $\delta$ (ppm): 103.8 (C-1'), 84.9 ( ( $\left.\mathrm{C}_{\mathrm{pss}}\right), 83.4$ (C-1), 80.7, 78.9, 76.5 (C-3, 4, 5), 75.5 (C-5'), 73.2 (C-2'), 72.9 (C-2), 70.5 (C-4'), 69.6, 69.2, 68.7, $68.2\left(\mathrm{C}_{\mathrm{Cp}}\right), 68.1$ (C-3'), 60.6 (C-6), 60.4 (C-6'), $27.9\left(\mathrm{CH}_{2} \mathrm{~S}\right)$; HMRS $(\mathrm{FAB}+)$ : Calc. for $\mathrm{C}_{36} \mathrm{H}_{54} \mathrm{O}_{20} \mathrm{~S}_{2} \mathrm{Fe} 926.1999$. Found $949.1901[\mathrm{M}+\mathrm{Na}]^{+}$.

General procedure for the synthesis of the azido-ferrocene derivatives 18 and 19. A solution of ferrocene derivative $\mathbf{1}(50 \mathrm{mg}, 0.231 \mathrm{mmol})$ or $\mathbf{8}(50 \mathrm{mg}, 0.203 \mathrm{mmol})$ ) and sodium azide (6 equiv per hydroxyl group) in glacial acetic acid $(3 \mathrm{~mL})$ was stirred at $50{ }^{\circ} \mathrm{C}$ for $3 \mathrm{~h}$. The reaction mixture was diluted in $\mathrm{CH}_{2} \mathrm{Cl}_{2}(50 \mathrm{~mL})$ and the organic phase was washed with a saturated solution of $\mathrm{NaHCO}_{3}(3 \mathrm{x}$ $50 \mathrm{~mL})$, dried $\left(\mathrm{Na}_{2} \mathrm{SO}_{4}\right)$, filtered and evaporated under vacuum. The crude was purified by column chromatography.

Azidomethylferrocene (18). Column chromatography (EtOAc-Hexane 1:25) gave 18 (51 mg, 92\%) as a dark red oil: IR (Film, $\left.\mathrm{cm}^{-1}\right)$ : 3093, 2922, 2853, 2090; ${ }^{1} \mathrm{H}-\mathrm{NMR}\left(300 \mathrm{MHz}, \mathrm{CDCl}_{3}\right), \delta$ (ppm): $4.23\left(\mathrm{~m}, 2 \mathrm{H}, \mathrm{H}_{\mathrm{Cp}}\right), 4.19$ (m, 2H, $\left.\mathrm{H}_{\mathrm{Cp}}\right), 4.16$ (bs, 5H, $\mathrm{H}_{\mathrm{Cp}}$ ), 4.11 (bs, $\left.2 \mathrm{H}, \mathrm{CH}_{2} \mathrm{~N}_{3}\right) ;{ }^{13} \mathrm{C}-\mathrm{NMR}(75 \mathrm{MHz}$, $\left.\mathrm{CDCl}_{3}\right), \delta(\mathrm{ppm}): 82.2\left(\mathrm{C}_{\mathrm{ipso}}\right), 68.8\left(\mathrm{C}_{\mathrm{Cp}} \mathrm{C}_{\mathrm{Cp}}\right), 68.6\left(\mathrm{C}_{\mathrm{Cp}}\right), 51.0\left(\mathrm{CH}_{2} \mathrm{~N}_{3}\right)$; HMRS (FAB+): Calc. for $\mathrm{C}_{11} \mathrm{H}_{11} \mathrm{~N}_{3} \mathrm{Fe} 241.0302$. Found 241.0302 $\mathrm{M}^{+}$.

1,1'-Bis(azidomethyl)ferrocene (19). Column chromatography (ether-Hexane 1:3) gave 19 (57 $\mathrm{mg}, 95 \%$ ) as a dark red oil: IR (Film, $\mathrm{cm}^{-1}$ ): 2967, 2921, 2852, 2085; ${ }^{1} \mathrm{H}-\mathrm{NMR}$ (300 MHz, $\mathrm{CDCl}_{3}$ ), $\delta$ (ppm): $4.22\left(\mathrm{~m}, 2 \mathrm{H}, \mathrm{H}_{\mathrm{Cp}}\right), 4.20\left(\mathrm{~m}, 2 \mathrm{H}, \mathrm{H}_{\mathrm{Cp}}\right), 4.11$ (bs, $\left.4 \mathrm{H}, \mathrm{CH}_{2} \mathrm{~N}_{3}\right) ;{ }^{13} \mathrm{C}-\mathrm{NMR}\left(75 \mathrm{MHz}, \mathrm{CDCl}_{3}\right), \delta$ (ppm): $80.2\left(\mathrm{C}_{\text {ipso }}\right), 69.5\left(\mathrm{C}_{\mathrm{Cp}}\right), 69,3\left(\mathrm{C}_{\mathrm{Cp}}\right), 50.7\left(\mathrm{CH}_{2} \mathrm{~N}_{3}\right)$; HMRS $(\mathrm{FAB}+)$ : Calc. for $\mathrm{C}_{12} \mathrm{H}_{12} \mathrm{~N}_{6} \mathrm{Fe}$ 296.0473. Found $296.0473 \mathrm{M}^{+}$.

General procedure for the synthesis of 1,2,3-triazol derivatives 22-25, 29 and 30. To a solution of azido-ferrocene derivative 18 or 19 and propargyl glicoside 20 or 21 (1.1 equiv per azido group), or 1-ethynylferrocene $\mathbf{2 6}$ and 2-azidoethyl glicoside $\mathbf{2 7}$ or $\mathbf{2 8}$ (1.1 equiv) in dry toluene (6 mL) was added $(\mathrm{EtO})_{3} \mathrm{P} \cdot \mathrm{CuI}(0.2$ equiv per azido group). The mixture was stirred under reflux for $45 \mathrm{~min}$. The solvent was removed by evaporation under vacuum and the crude was purified by column chromatography to yield the corresponding 1,2,3-triazol derivative. 


\section{[4-(2,3,4,6-Tetra- $O$-acetyl- $\beta$-D-glucopyranosyloxymethyl)-1 $H$-1,2,3-triazol-1-yl]methyl-}

ferrocene (22). Starting from 18 (40 $\mathrm{mg}, 0.166 \mathrm{mmol})$, column chromatography (EtOAc-Hexane 2:1) gave 22 (99 mg, 95\%) as a yellow solid: M. p. $51^{\circ} \mathrm{C}$; $[\alpha]_{\mathrm{D}}-24^{\circ}\left(c \mathrm{1}, \mathrm{CHCl}_{3}\right)$; IR ( $\left.\mathrm{KBr}, \mathrm{cm}^{-1}\right): 2952,2922$, 1750, 1373, 1224, 1039; ${ }^{1} \mathrm{H}-\mathrm{NMR}\left(300 \mathrm{MHz}, \mathrm{CDCl}_{3}\right), \delta(\mathrm{ppm}): 7.43$ (s, 1H, H-5-C $\left.\mathrm{HN}_{3}\right), 5.30$ (s, $2 \mathrm{H}$, $\left.\mathrm{CH}_{2} \mathrm{~N}\right), 5.18\left(\mathrm{t}, 1 \mathrm{H},{ }^{3} \mathrm{~J}=9.4 \mathrm{~Hz}, \mathrm{H}-3\right), 5.08\left(\mathrm{t}, 1 \mathrm{H},{ }^{3} \mathrm{~J}=9.6 \mathrm{~Hz}, \mathrm{H}-4\right), 4.98\left(\mathrm{t}, 1 \mathrm{H},{ }^{3} \mathrm{~J}=8.7 \mathrm{~Hz}, \mathrm{H}-2\right), 4.88$ $\left(\mathrm{d}, 1 \mathrm{H},{ }^{2} \mathrm{~J}=12.7 \mathrm{~Hz}, \mathrm{CH}_{2} \mathrm{O}\right), 4.79\left(\mathrm{~d}, 1 \mathrm{H},{ }^{2} \mathrm{~J}=12.7 \mathrm{~Hz}, \mathrm{CH}_{2} \mathrm{O}\right), 4.66\left(\mathrm{~d}, 1 \mathrm{H},{ }^{3} J_{1,2}=8.0 \mathrm{~Hz}, \mathrm{H}-1\right), 4.28(\mathrm{t}$, $\left.2 \mathrm{H},{ }^{3} J=1.8 \mathrm{~Hz}, \mathrm{H}_{\mathrm{Cp}}\right), 4.26\left(\mathrm{dd}, 1 \mathrm{H},{ }^{2} J_{6,6}=12.5 \mathrm{~Hz},{ }^{3} J_{5,6}=4.8 \mathrm{~Hz}, \mathrm{H}-6\right), 4.22\left(\mathrm{t}, 2 \mathrm{H},{ }^{3} J=1.8 \mathrm{~Hz}, \mathrm{H}_{\mathrm{Cp}}\right)$, $4.20\left(\mathrm{~s}, 5 \mathrm{H}, \mathrm{H}_{\mathrm{Cp}}\right), 4.12\left(\mathrm{dd}, 1 \mathrm{H},{ }^{2} J_{6,6^{\prime}}=12.4 \mathrm{~Hz},{ }^{3} J_{5,6^{\prime}}=2.3 \mathrm{~Hz}, \mathrm{H}-6\right.$ '), 3.71 (ddd, $1 \mathrm{H},{ }^{3} J_{4,5}=9.8 \mathrm{~Hz},{ }^{3} J_{5,6}$ $\left.=4.7 \mathrm{~Hz},{ }^{3} J_{5,6^{\prime}}=2.4 \mathrm{~Hz}, \mathrm{H}-5\right), 2.08\left(\mathrm{~s}, 3 \mathrm{H}, \mathrm{CH}_{3} \mathrm{CO}\right), 2.02$ (s, 3H, $\left.\mathrm{CH}_{3} \mathrm{CO}\right), 1.99$ (s, 3H, CH$\left.{ }_{3} \mathrm{CO}\right), 1.89$ (s, $\left.3 \mathrm{H}, \mathrm{CH}_{3} \mathrm{CO}\right) ;{ }^{13} \mathrm{C}-\mathrm{NMR}\left(75 \mathrm{MHz}, \mathrm{CDCl}_{3}\right), \delta(\mathrm{ppm}): 170.5,170.1,169.4(\mathrm{CO}), 144.0\left(\mathrm{C}-4-\mathrm{C}_{2} \mathrm{HN}_{3}\right), 122.1$ $\left(\mathrm{C}-5-\mathrm{C}_{2} \mathrm{HN}_{3}\right), 99.7(\mathrm{C}-1), 80.6\left(\mathrm{C}_{\mathrm{ipso}}\right), 72.8$ (C-3), $71.8(\mathrm{C}-5), 71.2(\mathrm{C}-2), 69.1,68.9\left(\mathrm{C}_{\mathrm{Cp}}, \mathrm{C}_{\mathrm{Cp}}\right), 68.3(\mathrm{C}-$ 4), $62.9\left(\mathrm{CH}_{2} \mathrm{O}\right), 61.8(\mathrm{C}-6), 50.1\left(\mathrm{CH}_{2} \mathrm{~N}\right), 20.7,20.6,20.5\left(\mathrm{CH}_{3} \mathrm{CO}\right)$; HMRS (FAB+): Calc. for $\mathrm{C}_{28} \mathrm{H}_{33} \mathrm{O}_{10} \mathrm{~N}_{3} \mathrm{Fe}$ 627.1515. Found 650.1410 [M+Na] $]^{+}$.

\section{[4-(2,3,4,6-Tetra-O-acetyl- $\alpha$-D-mannopyranosyloxymethyl)-1H-1,2,3-triazol-1-yl]methyl-}

ferrocene (23). Starting from $18(50 \mathrm{mg}, 0.207 \mathrm{mmol})$, column chromatography (EtOAc-Hexane 2:1) gave $23(127 \mathrm{mg}, 98 \%)$ as a yellow solid: M. p. $45^{\circ} \mathrm{C}$; $[\alpha]_{\mathrm{D}}+32^{\circ}\left(c 1, \mathrm{CHCl}_{3}\right)$; IR $\left(\mathrm{KBr}, \mathrm{cm}^{-1}\right): 2934$, 1748, 1370, 1225, 1046; ${ }^{1} \mathrm{H}-\mathrm{NMR}\left(300 \mathrm{MHz}, \mathrm{CDCl}_{3}\right), \delta(\mathrm{ppm}): 7.48$ (s, 1H, H-5-C $\left.\mathrm{HN}_{3}\right), 5.30-5.22(\mathrm{~m}$, $\left.5 \mathrm{H}, \mathrm{CH}_{2} \mathrm{~N}, \mathrm{H}-2,3,4\right), 4.93$ (s, $\left.1 \mathrm{H}, \mathrm{H}-1\right), 4.78$ (d, $\left.1 \mathrm{H},{ }^{2} \mathrm{~J}=12.2 \mathrm{~Hz}, \mathrm{CH}_{2} \mathrm{O}\right), 4.64\left(\mathrm{~d}, 1 \mathrm{H},{ }^{2} \mathrm{~J}=12.2 \mathrm{~Hz}\right.$, $\left.\mathrm{CH}_{2} \mathrm{O}\right), 4.32\left(\mathrm{bs}, 2 \mathrm{H}, \mathrm{H}_{\mathrm{Cp}}\right), 4.29-4.16(\mathrm{~m}, 2 \mathrm{H}, \mathrm{H}-5,6), 4.24\left(\mathrm{bs}, 2 \mathrm{H}, \mathrm{H}_{\mathrm{Cp}}\right), 4.21\left(\mathrm{~s}, 5 \mathrm{H}, \mathrm{H}_{\mathrm{Cp}}\right), 4.05(\mathrm{bd}, 1 \mathrm{H}$, ${ }^{2} J_{6,6},=12.0 \mathrm{~Hz}, \mathrm{H}-6$ '), $2.14\left(\mathrm{~s}, 3 \mathrm{H}, \mathrm{CH}_{3} \mathrm{CO}\right), 2.10$ (s, 3H, $\left.\mathrm{CH}_{3} \mathrm{CO}\right), 2.02\left(\mathrm{~s}, 3 \mathrm{H}, \mathrm{CH}_{3} \mathrm{CO}\right), 1.97$ (s, 3H, $\left.\mathrm{CH}_{3} \mathrm{CO}\right) ;{ }^{13} \mathrm{C}-\mathrm{NMR}\left(75 \mathrm{MHz}, \mathrm{CDCl}_{3}\right), \delta(\mathrm{ppm}): 169.8(\mathrm{CO}), 143.3\left(\mathrm{C}-4-\mathrm{C}_{2} \mathrm{HN}_{3}\right), 122.3\left(\mathrm{C}-5-\mathrm{C}_{2} \mathrm{HN}_{3}\right)$, $96.9(\mathrm{C}-1), 80.7\left(\mathrm{C}_{\mathrm{ipso}}\right), 69.4,69.3,69.1,68.7\left(\mathrm{C}-2,3,5, \mathrm{C}_{\mathrm{Cp}}\right), 69.0\left(\mathrm{C}_{\mathrm{Cp}}\right), 66.1(\mathrm{C}-4), 62.3\left(\mathrm{CH}_{2} \mathrm{O}\right), 61.1$ (C-6), $50.2\left(\mathrm{CH}_{2} \mathrm{~N}\right), 20.9,20.8,20.7\left(\mathrm{CH}_{3} \mathrm{CO}\right)$; HMRS (FAB+): Calc. for $\mathrm{C}_{28} \mathrm{H}_{33} \mathrm{O}_{10} \mathrm{~N}_{3} \mathrm{Fe} 627.1515$. Found $650.1411[\mathrm{M}+\mathrm{Na}]^{+}$.

\section{1,1'-Bis $\{[4-(2,3,4,6$-tetra- $O$-acetyl- $\beta$-D-glucopyranosyloxymethyl)-1H-1,2,3-triazol-1-yl]me-}

thyl\}ferrocene (24). Starting from 19 (55 mg, $0.186 \mathrm{mmol}$ ), column chromatography (EtOAc-Hexane 4:1 $\rightarrow 6: 1)$ gave $24(176 \mathrm{mg}, 88 \%)$ as a yellow solid: M. p. $85^{\circ} \mathrm{C} ;[\alpha]_{\mathrm{D}}-26^{\circ}\left(c 1, \mathrm{CHCl}_{3}\right)$; IR $\left(\mathrm{KBr}, \mathrm{cm}^{-1}\right)$ : 2954, 2853, 1753, 1371, 1226, 1041; ${ }^{1} \mathrm{H}-\mathrm{NMR}$ (300 MHz, $\left.\mathrm{CDCl}_{3}\right), \delta(\mathrm{ppm}): 7.51$ (s, 2H, H-5-C $\mathrm{HN}_{3}$ ), $5.26\left(\mathrm{~s}, 4 \mathrm{H}, \mathrm{CH}_{2} \mathrm{~N}\right), 5.19$ (t, 2H, $\left.{ }^{3} \mathrm{~J}=9.4 \mathrm{~Hz}, \mathrm{H}-3\right), 5.08$ (t, 2H, $\left.{ }^{3} \mathrm{~J}=9.6 \mathrm{~Hz}, \mathrm{H}-4\right), 4.98\left(\mathrm{t}, 2 \mathrm{H},{ }^{3} \mathrm{~J}=8.7 \mathrm{~Hz}\right.$, $\mathrm{H}-2), 4.92\left(\mathrm{~d}, 2 \mathrm{H},{ }^{2} J=12.6 \mathrm{~Hz}, \mathrm{CH}_{2} \mathrm{O}\right), 4.79\left(\mathrm{~d}, 2 \mathrm{H},{ }^{2} J=12.5 \mathrm{~Hz}, \mathrm{CH}_{2} \mathrm{O}\right), 4.68\left(\mathrm{~d}, 2 \mathrm{H},{ }^{3} J_{1,2}=7.9 \mathrm{~Hz}, \mathrm{H}-\right.$ 1), 4.28 (bs, $4 \mathrm{H}, \mathrm{H}_{\mathrm{Cp}}$ ), 4.23 (bs, 6H, $\left.\mathrm{H}_{\mathrm{Cp}}, \mathrm{H}-6\right), 4.14$ (dd, $2 \mathrm{H},{ }^{2} J_{6,6},=12.2 \mathrm{~Hz},{ }^{3} J_{5,6}$, = $2.2 \mathrm{~Hz}, \mathrm{H}-6$ '), 3.74 $\left(\mathrm{ddd}, 2 \mathrm{H},{ }^{3} J_{4,5}=9.7 \mathrm{~Hz},{ }^{3} J_{5,6}=4.0 \mathrm{~Hz},{ }^{3} J_{5,6}=2.2 \mathrm{~Hz}, \mathrm{H}-5\right), 2.08\left(\mathrm{~s}, 6 \mathrm{H}, \mathrm{CH}_{3} \mathrm{CO}\right), 2.02\left(\mathrm{~s}, 6 \mathrm{H}, \mathrm{CH}_{3} \mathrm{CO}\right)$, 1.98 (s, 6H, $\left.\mathrm{CH}_{3} \mathrm{CO}\right), 1.91$ (s, 6H, $\left.\mathrm{CH}_{3} \mathrm{CO}\right) ;{ }^{13} \mathrm{C}-\mathrm{NMR}\left(75 \mathrm{MHz}, \mathrm{CDCl}_{3}\right.$ ), $\delta$ (ppm): 170.5, 170.0, 169.3, 169.2 (CO), $144.1\left(\mathrm{C}-4-\mathrm{C}_{2} \mathrm{HN}_{3}\right), 122.5\left(\mathrm{C}-5-\mathrm{C}_{2} \mathrm{HN}_{3}\right), 99.9(\mathrm{C}-1), 82.3\left(\mathrm{C}_{\mathrm{ipso}}\right), 72.7$ (C-3), $71.8(\mathrm{C}-5), 71.1$ $(\mathrm{C}-2), 69.9,69.8,69.6,69.4\left(\mathrm{C}_{\mathrm{Cp}}\right), 68.3(\mathrm{C}-4), 69.2\left(\mathrm{CH}_{2} \mathrm{O}\right), 61.7(\mathrm{C}-6), 49.4\left(\mathrm{CH}_{2} \mathrm{~N}\right), 20.7,20.6,20.5$ $\left(\mathrm{CH}_{3} \mathrm{CO}\right)$; MALDI-TOF: Calc. for $\mathrm{C}_{46} \mathrm{H}_{56} \mathrm{O}_{20} \mathrm{~N}_{6} \mathrm{Fe}$ 1068.290. Found 1068.171 [M] $]^{+}, 1069.184[\mathrm{M}+\mathrm{H}]^{+}$, $1091.285[\mathrm{M}+\mathrm{Na}]^{+}$. 
1,1'-Bis\{[4-(2,3,4,6-tetra- $O$-acetyl- $\alpha$-D-mannopyranosyloxymethyl)-1 $H$-1,2,3-triazol-1-yl]-

methyl\}ferrocene (25). Starting from 19 (50 mg, $0.169 \mathrm{mmol}$ ), column chromatography (EtOAc-Hexane 10:1) gave 25 (171 mg, 94\%) as a yellow solid: M. p. $79^{\circ} \mathrm{C}$; $[\alpha]_{\mathrm{D}}+39^{\circ}\left(\mathrm{c} 1, \mathrm{CHCl}_{3}\right)$; IR $\left(\mathrm{KBr}, \mathrm{cm}^{-1}\right)$ :

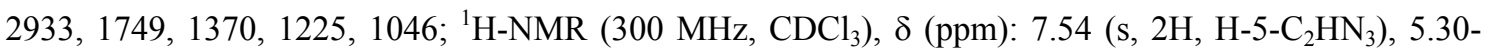
5.29 (m, 8H, $\left.\mathrm{CH}_{2} \mathrm{~N}, \mathrm{H}-2,4\right), 5.22$ (bs, $2 \mathrm{H}, \mathrm{H}-3$ ), 4.95 (s, $\left.2 \mathrm{H}, \mathrm{H}-1\right), 4.82$ (d, $2 \mathrm{H},{ }^{2} J=12.3 \mathrm{~Hz}, \mathrm{CH}_{2} \mathrm{O}$ ), 4.66 (d, $2 \mathrm{H},{ }^{2} J=12.3 \mathrm{~Hz}, \mathrm{CH}_{2} \mathrm{O}$ ), 4.31 (bs, 4H, $\mathrm{H}_{\mathrm{Cp}}$ ), 4.30-4.25 (m, 6H, $\mathrm{H}_{\mathrm{Cp}}, \mathrm{H}-6$ ), 4.13-4.03 (m, 4H, H-5, 6'), 2.14 (s, 6H, $\mathrm{CH}_{3} \mathrm{CO}$ ), 2.11 (s, 6H, $\mathrm{CH}_{3} \mathrm{CO}$ ), 2.03 (s, 6H, $\mathrm{CH}_{3} \mathrm{CO}$ ), 1.97 (s, 6H, $\mathrm{CH}_{3} \mathrm{CO}$ ); ${ }^{13} \mathrm{C}-\mathrm{NMR}(75$ $\left.\mathrm{MHz}, \mathrm{CDCl}_{3}\right), \delta(\mathrm{ppm}):$ 170.0, $169.7(\mathrm{CO}), 143.5\left(\mathrm{C}-4-\mathrm{C}_{2} \mathrm{HN}_{3}\right), 122.6\left(\mathrm{C}-5-\mathrm{C}_{2} \mathrm{HN}_{3}\right), 97.0(\mathrm{C}-1), 82.2$ $\left(\mathrm{C}_{\mathrm{ipso}}\right), 70.1,69.7\left(\mathrm{C}_{\mathrm{Cp}}\right), 69.5,69.1,68.7(\mathrm{C}-2,3,5), 66.1(\mathrm{C}-4), 62.4\left(\mathrm{CH}_{2} \mathrm{O}\right), 61.1(\mathrm{C}-6), 49.7\left(\mathrm{CH}_{2} \mathrm{~N}\right)$, 20.9, 20.8, $20.7\left(\mathrm{CH}_{3} \mathrm{CO}\right)$; MALDI-TOF: Calc. for $\mathrm{C}_{46} \mathrm{H}_{56} \mathrm{O}_{20} \mathrm{~N}_{6} \mathrm{Fe}$ 1068.290. Found 1091.277 [M+ Na] $]^{+}$

\section{[1-(2,3,4,6-Tetra- $O$-acetyl- $\beta$-D-glucopyranosyloxyethyl)-1H-1,2,3-triazol-4-yl]ferrocene}

(29). Starting from 26 (20 mg, $0.095 \mathrm{mmol}$ ), column chromatography (EtOAc-Hexane 2:1) gave 29 (57 mg, $95 \%)$ as an orange solid: M. p. $184{ }^{\circ} \mathrm{C} ;[\alpha]_{\mathrm{D}}-56^{\circ}\left(c 1, \mathrm{CHCl}_{3}\right)$; IR $\left(\mathrm{KBr}, \mathrm{cm}^{-1}\right): 2953,1750,1372$,

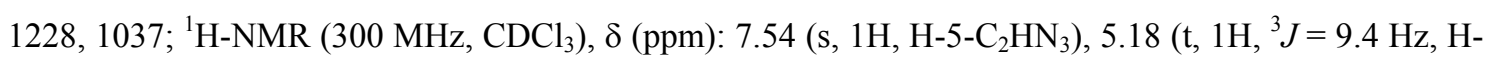
3), 5.07 (t, $\left.1 \mathrm{H},{ }^{3} J=9.5 \mathrm{~Hz}, \mathrm{H}-4\right), 5.03\left(\mathrm{t}, 1 \mathrm{H},{ }^{3} J=8.7 \mathrm{~Hz}, \mathrm{H}-2\right), 4.82$ (bs, $1 \mathrm{H}, \mathrm{H}_{\mathrm{Cp}}$ ), 4.68 (bs, $1 \mathrm{H}, \mathrm{H}_{\mathrm{Cp}}$ ), 4.68-4.60 (m, $\left.1 \mathrm{H}, \mathrm{CH}_{2} \mathrm{~N}\right), 4.52\left(\mathrm{ddd}, 1 \mathrm{H},{ }^{2} J=14.5 \mathrm{~Hz},{ }^{3} J=8.4 \mathrm{~Hz},{ }^{3} J=3.5 \mathrm{~Hz}, \mathrm{CH}_{2} \mathrm{~N}\right), 4.47\left(\mathrm{~d}, 1 \mathrm{H},{ }^{3} J_{1,2}\right.$ $=7.7 \mathrm{~Hz}, \mathrm{H}-1), 4.29\left(\mathrm{bs}, 2 \mathrm{H}, \mathrm{H}_{\mathrm{Cp}}\right), 4.29-4.22\left(\mathrm{~m}, 2 \mathrm{H}, \mathrm{H}-6, \mathrm{CH}_{2} \mathrm{O}\right), 4.14\left(\mathrm{dd}, 1 \mathrm{H},{ }^{2} J_{6,6},=12.6 \mathrm{~Hz},{ }^{3} J_{5,6}{ }=\right.$ $2.4 \mathrm{~Hz}, \mathrm{H}-6$ '), 4.09 (bs, $5 \mathrm{H}, \mathrm{H}_{\mathrm{Cp}}$ ), $3.90\left(\mathrm{~m}, 1 \mathrm{H},{ }^{3} \mathrm{~J}=3.3 \mathrm{~Hz}, \mathrm{CH}_{2} \mathrm{O}\right.$ ), 3.70 (ddd, $1 \mathrm{H},{ }^{3} J_{4,5}=9.8 \mathrm{~Hz},{ }^{3} J_{5,6}=$ $\left.4.7 \mathrm{~Hz},{ }^{3} J_{5,6}=2.3 \mathrm{~Hz}, \mathrm{H}-5\right), 2.08$ (s, $3 \mathrm{H}, \mathrm{CH}_{3} \mathrm{CO}$ ), 2.02 (s, 3H, $\mathrm{CH}_{3} \mathrm{CO}$ ), 1.99 (s, 3H, $\mathrm{CH}_{3} \mathrm{CO}$ ), 1.84 (s, $\left.3 \mathrm{H}, \mathrm{CH}_{3} \mathrm{CO}\right) ;{ }^{13} \mathrm{C}-\mathrm{NMR}\left(75 \mathrm{MHz}, \mathrm{CDCl}_{3}\right), \delta(\mathrm{ppm}): 170.1,169.5,169.4(\mathrm{CO}), 146.8\left(\mathrm{C}-4-\mathrm{C}_{2} \mathrm{HN}_{3}\right), 120.5$ $\left(\mathrm{C}-5-\mathrm{C}_{2} \mathrm{HN}_{3}\right), 100.6(\mathrm{C}-1), 75.5\left(\mathrm{C}_{\mathrm{ipso}}\right), 72.5(\mathrm{C}-3), 72.1(\mathrm{C}-2), 71.0(\mathrm{C}-5), 69.6\left(\mathrm{C}_{\mathrm{Cp}}\right), 68.7\left(\mathrm{C}_{\mathrm{Cp}}\right), 68.6$ $\left(\mathrm{C}_{\mathrm{Cp}}\right), 68.3(\mathrm{C}-4), 67.9\left(\mathrm{CH}_{2} \mathrm{O}\right), 66.7\left(\mathrm{C}_{\mathrm{Cp}}\right), 66.6\left(\mathrm{C}_{\mathrm{Cp}}\right), 61.8(\mathrm{C}-6), 50.0\left(\mathrm{CH}_{2} \mathrm{~N}\right), 20.8,20.6\left(\mathrm{CH}_{3} \mathrm{CO}\right)$; HMRS (FAB+): Calc. for $\mathrm{C}_{28} \mathrm{H}_{33} \mathrm{O}_{10} \mathrm{~N}_{3} \mathrm{Fe}$ 627.1515. Found $650.1420[\mathrm{M}+\mathrm{Na}]^{+}$.

\section{[1-(2,3,4,6-Tetra- $O$-acetyl- $\alpha$-D-mannopyranosyloxyethyl)-1 $H$-1,2,3-triazol-4-yl]ferrocene}

(30). Starting from 26 (50 mg, $0.238 \mathrm{mmol}$ ), column chromatography (EtOAc-Hexane 2:1) gave 30 (146 $\mathrm{mg}, 98 \%)$ as an orange solid: M. p. $66^{\circ} \mathrm{C} ;[\alpha]_{\mathrm{D}}-13^{\circ}\left(c 1, \mathrm{CHCl}_{3}\right)$; IR $\left(\mathrm{KBr}, \mathrm{cm}^{-1}\right): 2954,2852,1748$, 1370, 1224, 1046; ${ }^{1} \mathrm{H}-\mathrm{NMR}\left(300 \mathrm{MHz}, \mathrm{CDCl}_{3}\right.$ ), $\delta$ (ppm): 7.64 (s, 1H, H-5- $\mathrm{C}_{2} \mathrm{HN}_{3}$ ), 5.26 (bs, 3H, H-2, 3, 4), 4.84 (s, $1 \mathrm{H}, \mathrm{H}-1), 4.80$ (bs, $\left.1 \mathrm{H}, \mathrm{H}_{\mathrm{Cp}}\right), 4.74$ (bs, $1 \mathrm{H}, \mathrm{H}_{\mathrm{Cp}}$ ), $4.61\left(\mathrm{~m}, 2 \mathrm{H}, \mathrm{CH}_{2} \mathrm{~N}\right), 4.29$ (bs, $2 \mathrm{H}, \mathrm{H}_{\mathrm{Cp}}$ ), 4.20-4.11 (m, $\left.1 \mathrm{H}, \mathrm{CH}_{2} \mathrm{O}\right), 4.16\left(\mathrm{dd}, 1 \mathrm{H},{ }^{2} J_{6,6^{\prime}}=12.2 \mathrm{~Hz},{ }^{3} J_{5,6}=4.9 \mathrm{~Hz}, \mathrm{H}-6\right), 4.07$ (bs, 5H, $\mathrm{H}_{\mathrm{Cp}}$ ), 3.99 $\left(\mathrm{dd}, 1 \mathrm{H},{ }^{2} J_{6,6^{\prime}}=12.3 \mathrm{~Hz},{ }^{3} J_{5,6^{\prime}}=2.1 \mathrm{~Hz}, \mathrm{H}-6^{\prime}\right), 3.87$ (ddd, $1 \mathrm{H},{ }^{2} J=9.7 \mathrm{~Hz},{ }^{3} J=4.2 \mathrm{~Hz},{ }^{3} J=4.2 \mathrm{~Hz}$, $\mathrm{CH}_{2} \mathrm{O}$ ), 3.43 (bs, $\left.1 \mathrm{H}, \mathrm{H}-5\right), 2.14$ (s, 3H, $\mathrm{CH}_{3} \mathrm{CO}$ ), 2.08 (s, 3H, $\mathrm{CH}_{3} \mathrm{CO}$ ), 2.01 (s, 3H, $\mathrm{CH}_{3} \mathrm{CO}$ ), 1.88 (s, $3 \mathrm{H}$, $\left.\mathrm{CH}_{3} \mathrm{CO}\right) ;{ }^{13} \mathrm{C}-\mathrm{NMR}\left(75 \mathrm{MHz}, \mathrm{CDCl}_{3}\right), \delta(\mathrm{ppm}): 170.5,170.0,169.7(\mathrm{CO}), 147.1\left(\mathrm{C}-4-\mathrm{C}_{2} \mathrm{HN}_{3}\right), 120.1(\mathrm{C}-$ 5- $\left.\mathrm{C}_{2} \mathrm{HN}_{3}\right), 97.4(\mathrm{C}-1), 75.4\left(\mathrm{C}_{\mathrm{ipso}}\right), 69.7\left(\mathrm{C}_{\mathrm{Cp}}\right), 69.3,69.1,68.9(\mathrm{C}-2,3,5), 68.7\left(\mathrm{C}_{\mathrm{Cp}_{\mathrm{p}}}\right), 66.8\left(\mathrm{C}_{\mathrm{Cp}}\right), 66.2$ $\left(\mathrm{CH}_{2} \mathrm{O}\right), 65.6(\mathrm{C}-4), 62.2(\mathrm{C}-6), 49.7\left(\mathrm{CH}_{2} \mathrm{~N}\right), 20.9,20.8,20.7,20.6\left(\mathrm{CH}_{3} \mathrm{CO}\right)$; HMRS (FAB+): Calc. for $\mathrm{C}_{28} \mathrm{H}_{33} \mathrm{O}_{10} \mathrm{~N}_{3} \mathrm{Fe}$ 627.1515. Found $650.1414[\mathrm{M}+\mathrm{Na}]^{+}$.

General procedure for the Zemplén de- $O$-acetylation of 1,2,3-triazol derivatives 22-25, 29 and 30. A solution of 1,2,3-triazol derivative 22-25 or $\mathbf{2 9}$ or $\mathbf{3 0}$ in dry $\mathrm{MeOH}(15-20 \mathrm{~mL})$ or in a mixture 
of dry $\mathrm{MeOH}$ and dry $\mathrm{CH}_{2} \mathrm{Cl}_{2}$ 10:1 (15-20 mL) was made alkaline to $\mathrm{pH}$ 9-10 (indicator paper) with a fresh methanolic solution of $\mathrm{NaOMe}(1 \mathrm{M})$ and stirred at room temperature for 2-24 $\mathrm{h}$ following the reaction by TLC. If the product precipitated, the solvent was concentrated under vacuum to $1-2 \mathrm{~mL}$ and ether was added. The solid was filtered off and washed with ether. In other case, the solvent was removed under vacuum and the crude was purified by column chromatography. The pure compound was dissolved in water and lyophilised.

[4-( $\beta$-D-Glucopyranosyloxymethyl)-1H-1,2,3-triazol-1-yl]methylferrocene (31). Starting from 22 (306 mg, $0.488 \mathrm{mmol}$ ) in dry $\mathrm{MeOH}$, column chromatography (EtOAc-MeOH 3:2) gave 31 (213 mg, 95\%) as a yellow solid: M. p. $66.0^{\circ} \mathrm{C} ;[\alpha]_{\mathrm{D}}-22^{\circ}(c \mathrm{c} 25, \mathrm{MeOH})$; IR $\left(\mathrm{KBr}, \mathrm{cm}^{-1}\right): 3379,2883,1059$, 1022; ${ }^{1} \mathrm{H}-\mathrm{NMR}\left(300 \mathrm{MHz}, \mathrm{D}_{2} \mathrm{O}, 60{ }^{\circ} \mathrm{C}\right), \delta(\mathrm{ppm}): 8.39$ (s, 1H, H-5- $\left.\mathrm{C}_{2} \mathrm{HN}_{3}\right), 5.72\left(\mathrm{~s}, 2 \mathrm{H}, \mathrm{CH}_{2} \mathrm{~N}\right), 5.30(\mathrm{~d}$, $\left.1 \mathrm{H},{ }^{2} \mathrm{~J}=12.8 \mathrm{~Hz}, \mathrm{CH}_{2} \mathrm{O}\right), 5.22\left(\mathrm{~d}, 1 \mathrm{H},{ }^{2} \mathrm{~J}=12.6 \mathrm{~Hz}, \mathrm{CH}_{2} \mathrm{O}\right), 4.86\left(\mathrm{~d}, 1 \mathrm{H},{ }^{3} J_{1,2}=8.1 \mathrm{~Hz}, \mathrm{H}-1\right), 4.75(\mathrm{t}, 2 \mathrm{H}$, $\left.{ }^{3} J=1.3 \mathrm{~Hz}, \mathrm{H}_{\mathrm{Cp}}\right), 4.66\left(\mathrm{t}, 2 \mathrm{H},{ }^{3} J=1.3 \mathrm{~Hz}, \mathrm{H}_{\mathrm{Cp}}\right), 4.61\left(\mathrm{bs}, 5 \mathrm{H}, \mathrm{H}_{\mathrm{Cp}}\right), 4.21\left(\mathrm{dd}, 1 \mathrm{H},{ }^{2} J_{6,6},=12.5 \mathrm{~Hz},{ }^{3} J_{5,6}=\right.$ $1.5 \mathrm{~Hz}, \mathrm{H}-6), 4.05$ (dd, $1 \mathrm{H},{ }^{2} J_{6,6^{\prime}}=12.5 \mathrm{~Hz},{ }^{3} J_{5,6^{\prime}}=5.3 \mathrm{~Hz}, \mathrm{H}-6$ '), 3.82 (t, $\left.1 \mathrm{H},{ }^{3} J=9.1 \mathrm{~Hz}, \mathrm{H}-3\right), 3.74$ (m, $1 \mathrm{H}, \mathrm{H}-4), 3.70$ (t, $\left.1 \mathrm{H},{ }^{3} \mathrm{~J}=1.5 \mathrm{~Hz}, \mathrm{H}-5\right), 3.64$ (t, $\left.1 \mathrm{H},{ }^{3} \mathrm{~J}=8.3 \mathrm{~Hz}, \mathrm{H}-2\right) ;{ }^{13} \mathrm{C}-\mathrm{NMR}\left(75 \mathrm{MHz}, \mathrm{D}_{2} \mathrm{O}, 60{ }^{\circ} \mathrm{C}\right)$, $\delta(\mathrm{ppm}): 144.1\left(\mathrm{C}-4-\mathrm{C}_{2} \mathrm{HN}_{3}\right), 125.3\left(\mathrm{C}-5-\mathrm{C}_{2} \mathrm{HN}_{3}\right), 101.9(\mathrm{C}-1), 82.2\left(\mathrm{C}_{\mathrm{ipso}}\right), 76.4(\mathrm{C}-5), 76.3(\mathrm{C}-3), 73.5$ (C-2), $70.1(\mathrm{C}-4), 69.4\left(\mathrm{C}_{\mathrm{Cp}}, \mathrm{C}_{\mathrm{Cp}}\right), 69.2\left(\mathrm{C}_{\mathrm{Cp}}\right), 62.4\left(\mathrm{CH}_{2} \mathrm{O}\right), 61.3(\mathrm{C}-6), 50.2\left(\mathrm{CH}_{2} \mathrm{~N}\right)$; HMRS $(\mathrm{FAB}+)$ : Calc. for $\mathrm{C}_{20} \mathrm{H}_{25} \mathrm{O}_{6} \mathrm{~N}_{3} \mathrm{Fe} 459.1093$. Found $482.0990[\mathrm{M}+\mathrm{Na}]^{+}$.

[4-( $\alpha$-D-Mannopyranosyloxymethyl)-1H-1,2,3-triazol-1-yl]methylferrocene (32). Starting from 23 (201 mg, $0.320 \mathrm{mmol}$ ) in dry $\mathrm{MeOH}$, column chromatography (EtOAc-MeOH 3:2) gave 32 (139 mg, 95\%) as a yellow solid: M. p. $66^{\circ} \mathrm{C} ;[\alpha]_{\mathrm{D}}+56^{\circ}(c$ 0.25, $\mathrm{MeOH})$; IR $\left(\mathrm{KBr}, \mathrm{cm}^{-1}\right)$ : 3398, 2923, 1053; ${ }^{1} \mathrm{H}-\mathrm{NMR}\left(300 \mathrm{MHz}, \mathrm{CD}_{3} \mathrm{OD}\right), \delta(\mathrm{ppm}): 7.94$ (s, 1H, H-5- $\left.\mathrm{C}_{2} \mathrm{HN}_{3}\right), 5.34\left(\mathrm{~s}, 2 \mathrm{H}, \mathrm{CH}_{2} \mathrm{~N}\right), 4.85\left(\mathrm{~d}, 1 \mathrm{H},{ }^{3} J_{1,2}=\right.$ $10.1 \mathrm{~Hz}, \mathrm{H}-1), 4.76\left(\mathrm{~d}, 1 \mathrm{H},{ }^{2} J=12.3 \mathrm{~Hz}, \mathrm{CH}_{2} \mathrm{O}\right), 4.61\left(\mathrm{~d}, 1 \mathrm{H},{ }^{2} J=12.3 \mathrm{~Hz}, \mathrm{CH}_{2} \mathrm{O}\right), 4.34\left(\mathrm{~d}, 2 \mathrm{H},{ }^{3} J=1.6\right.$ $\mathrm{Hz}, \mathrm{H}_{\mathrm{Cp}}$ ), $4.22\left(\mathrm{t}, 2 \mathrm{H},{ }^{3} \mathrm{~J}=1.7 \mathrm{~Hz}, \mathrm{H}_{\mathrm{Cp}}\right.$ ), 4.19 (s, 5H, $\mathrm{H}_{\mathrm{Cp}}$ ), 3.82 (d, 1H, $\left.{ }^{2} J_{6,6},=11.7 \mathrm{~Hz}, \mathrm{H}-6\right), 3.77$ (bs, $1 \mathrm{H}, \mathrm{H}-2), 3.70\left(\mathrm{dd}, 1 \mathrm{H},{ }^{2} J_{6,6},=12.1 \mathrm{~Hz},{ }^{3} J_{5,6^{\prime}}=5.6 \mathrm{~Hz}, \mathrm{H}-6\right.$ '), $3.64\left(\mathrm{bd}, 1 \mathrm{H},{ }^{3} J=3.7 \mathrm{~Hz}, \mathrm{H}-3\right), 3.60(\mathrm{t}, 1 \mathrm{H}$, $\left.{ }^{3} J=9.1 \mathrm{~Hz}, \mathrm{H}-4\right), 3.56-3.52$ (m, 1H, H-5); ${ }^{13} \mathrm{C}-\mathrm{NMR}\left(75 \mathrm{MHz}, \mathrm{CD}_{3} \mathrm{OD}\right), \delta(\mathrm{ppm}): 145.2\left(\mathrm{C}-4-\mathrm{C}_{2} \mathrm{HN}_{3}\right)$, $124.8\left(\mathrm{C}-5-\mathrm{C}_{2} \mathrm{HN}_{3}\right), 100.8(\mathrm{C}-1), 83.0\left(\mathrm{C}_{\mathrm{ipso}}\right), 74.9(\mathrm{C}-5), 72.5(\mathrm{C}-3), 72.0(\mathrm{C}-2), 69.9\left(\mathrm{C}_{\mathrm{Cp}}\right), 69.8\left(\mathrm{C}_{\mathrm{Cp}}\right.$, $\mathrm{C}_{\mathrm{Cp}^{\prime}}$ ), 68.6 (C-4), $62.9(\mathrm{C}-6), 60.7\left(\mathrm{CH}_{2} \mathrm{O}\right), 51.0\left(\mathrm{CH}_{2} \mathrm{~N}\right)$; HMRS (FAB+): Calc. for $\mathrm{C}_{20} \mathrm{H}_{25} \mathrm{O}_{6} \mathrm{~N}_{3} \mathrm{Fe}$ 459.1093. Found $482.0989[\mathrm{M}+\mathrm{Na}]^{+}$.

\section{1,1'-Bis $\{[4-(\beta-D-g l u c o p y r a n o s y l o x y m e t h y l)-1 H-1,2,3-t r i a z o l-1-y l] m e t h y l\}$ ferrocene}

(33).

Starting from 24 (162 mg, $0.152 \mathrm{mmol})$ in dry MeOH, column chromatography (EtOAc-MeOH 2:1 $\rightarrow$ 1:1) gave 33 (103 mg, 93\%) as a yellow solid: M. p. $88^{\circ} \mathrm{C} ;[\alpha]_{\mathrm{D}}-28^{\circ}(c 0.25, \mathrm{MeOH})$; IR $\left(\mathrm{KBr}, \mathrm{cm}^{-1}\right)$ : 3404, 2923, 2883, 1052; ${ }^{1} \mathrm{H}-\mathrm{NMR}\left(300 \mathrm{MHz}, \mathrm{D}_{2} \mathrm{O}, 60{ }^{\circ} \mathrm{C}\right), \delta(\mathrm{ppm}): 8.39$ (s, 2H, H-5-C $\left.\mathrm{HN}_{3}\right), 5.69$ (s, $\left.4 \mathrm{H}, \mathrm{CH}_{2} \mathrm{~N}\right), 5.31\left(\mathrm{~d}, 2 \mathrm{H},{ }^{2} \mathrm{~J}=12.6 \mathrm{~Hz}, \mathrm{CH}_{2} \mathrm{O}\right), 5.22\left(\mathrm{~d}, 2 \mathrm{H},{ }^{2} \mathrm{~J}=12.7 \mathrm{~Hz}, \mathrm{CH}_{2} \mathrm{O}\right), 4.89\left(\mathrm{~d}, 2 \mathrm{H},{ }^{3} J_{1,2}=8.1\right.$ $\mathrm{Hz}, \mathrm{H}-1), 4.72$ (s, $\left.\mathrm{H}_{\mathrm{Cp}}, \mathrm{HDO}\right), 4.66$ (bs, $4 \mathrm{H}, \mathrm{H}_{\mathrm{Cp}}$ ), 4.22 (dd, $\left.2 \mathrm{H},{ }^{2} J_{6,6}=12.3 \mathrm{~Hz},{ }^{3} J_{5,6}=1.4 \mathrm{~Hz}, \mathrm{H}-6\right), 4.06$ $\left(\mathrm{dd}, 2 \mathrm{H},{ }^{2} J_{6,6^{\prime}}=12.3 \mathrm{~Hz},{ }^{3} J_{5,6^{\prime}}=5.0 \mathrm{~Hz}, \mathrm{H}-6\right.$ ') 3.83 (t, $2 \mathrm{H},{ }^{3} \mathrm{~J}=8.8 \mathrm{~Hz}, \mathrm{H}-3$ ), 3.76 (bs, 2H, H-4), 3.71 (dd, $\left.2 \mathrm{H},{ }^{3} J_{4,5}=1.9 \mathrm{~Hz},{ }^{3} J_{5,6}=1.5 \mathrm{~Hz}, \mathrm{H}-5\right), 3.64\left(\mathrm{t}, 2 \mathrm{H},{ }^{3} \mathrm{~J}=8.4 \mathrm{~Hz}, \mathrm{H}-2\right) ;{ }^{13} \mathrm{C}-\mathrm{NMR}\left(75 \mathrm{MHz}, \mathrm{D}_{2} \mathrm{O}, 60{ }^{\circ} \mathrm{C}\right), \delta$ (ppm): $144.2\left(\mathrm{C}-4-\mathrm{C}_{2} \mathrm{HN}_{3}\right), 125.3\left(\mathrm{C}-5-\mathrm{C}_{2} \mathrm{HN}_{3}\right), 102.0(\mathrm{C}-1), 82.9\left(\mathrm{C}_{\mathrm{ipso}}\right), 76.4(\mathrm{C}-5), 76.3(\mathrm{C}-2), 73.5(\mathrm{C}-$ 
3), $70.3\left(\mathrm{C}_{\mathrm{Cp}}\right), 70.1(\mathrm{C}-4), 70.0\left(\mathrm{C}_{\mathrm{Cp}}\right), 62.4\left(\mathrm{CH}_{2} \mathrm{O}\right), 61.3(\mathrm{C}-6), 49.9\left(\mathrm{CH}_{2} \mathrm{~N}\right)$; HMRS (FAB+): Calc. for $\mathrm{C}_{30} \mathrm{H}_{40} \mathrm{O}_{12} \mathrm{~N}_{6} \mathrm{Fe} 732.2054$. Found $755.1941[\mathrm{M}+\mathrm{Na}]^{+}$.

1,1'-Bis $\{[4-(\alpha-D-m a n n o p y r a n o s y l o x y m e t h y l)-1 H-1,2,3-t r i a z o l-1-y l] m e t h y l\}$ ferrocene $\quad$ (34). Starting from 25 (168 mg, $0.157 \mathrm{mmol}$ ) in dry $\mathrm{MeOH}$, column chromatography (EtOAc-MeOH 2:1 $\rightarrow$ 1:1) gave 34 (101 mg, 88\%) as a yellow solid: M. p. $103.0^{\circ} \mathrm{C} ;[\alpha]_{\mathrm{D}}+68^{\circ}(c 0.25, \mathrm{MeOH})$; $\mathrm{IR}\left(\mathrm{KBr}, \mathrm{cm}^{-1}\right)$ : 3409, 2926, 1058; ${ }^{1} \mathrm{H}-\mathrm{NMR}$ (300 MHz, $\mathrm{CD}_{3} \mathrm{OD}$ ), $\delta$ (ppm): 7.99 (s, 2H, H-5- $\mathrm{C}_{2} \mathrm{HN}_{3}$ ), 5.33 (bs, 4H, $\left.\mathrm{CH}_{2} \mathrm{~N}\right), 4.87\left(\mathrm{~d}, 2 \mathrm{H},{ }^{3} J_{1,2}=8.8 \mathrm{~Hz}, \mathrm{H}-1\right), 4.78\left(\mathrm{~d}, 2 \mathrm{H},{ }^{2} J=12.5 \mathrm{~Hz}, \mathrm{CH}_{2} \mathrm{O}\right), 4.63\left(\mathrm{~d}, 2 \mathrm{H},{ }^{2} J=12.3 \mathrm{~Hz}\right.$, $\mathrm{CH}_{2} \mathrm{O}$ ), 4.35 (bs, $4 \mathrm{H}, \mathrm{H}_{\mathrm{Cp}}$ ), 4.24 (bs, $4 \mathrm{H}, \mathrm{H}_{\mathrm{Cp}}$ ), 3.83 (bd, $2 \mathrm{H},{ }^{2} J_{6,6}=11.1 \mathrm{~Hz}, \mathrm{H}-6$ ), 3.78 (bs, 2H, H-2), $3.71\left(\mathrm{dd}, 2 \mathrm{H},{ }^{2} J_{6,6^{\prime}}=11.1 \mathrm{~Hz},{ }^{3} J_{5,6^{\prime}}=5.3 \mathrm{~Hz}, \mathrm{H}-6\right.$ '), 3.66 (bs, $\left.2 \mathrm{H}, \mathrm{H}-3\right), 3.62\left(\mathrm{t}, 2 \mathrm{H},{ }^{3} \mathrm{~J}=9.1 \mathrm{~Hz}, \mathrm{H}-4\right)$, 3.58-3.53 (m, 2H, H-5); ${ }^{13} \mathrm{C}-\mathrm{NMR}\left(75 \mathrm{MHz}, \mathrm{CD}_{3} \mathrm{OD}\right), \delta(\mathrm{ppm}): 145.2\left(\mathrm{C}-4-\mathrm{C}_{2} \mathrm{HN}_{3}\right), 125.1\left(\mathrm{C}-5-\mathrm{C}_{2} \mathrm{HN}_{3}\right)$, 100.7 (C-1), $84.1\left(\mathrm{C}_{\mathrm{ipso}}\right), 74.9(\mathrm{C}-5), 72.4(\mathrm{C}-3), 71.9(\mathrm{C}-2), 70.9\left(\mathrm{C}_{\mathrm{Cp}}\right), 70.8\left(\mathrm{C}_{\mathrm{Cp}}\right), 68.5(\mathrm{C}-4), 62.9(\mathrm{C}-6)$, $60.7\left(\mathrm{CH}_{2} \mathrm{O}\right), 50.6\left(\mathrm{CH}_{2} \mathrm{~N}\right)$; HMRS (FAB+): Calc. for $\mathrm{C}_{30} \mathrm{H}_{40} \mathrm{O}_{12} \mathrm{~N}_{6} \mathrm{Fe} 732.2054$. Found $755.1942[\mathrm{M}+$ $\mathrm{Na}]^{+}$.

[1-( $\beta$-D-Glucopyranosyloxyethyl)-1H-1,2,3-triazol-4-yl]ferrocene (35). Starting from 29 (222 $\mathrm{mg}, 0.354 \mathrm{mmol}$ ) in dry $\mathrm{MeOH}-\mathrm{CH}_{2} \mathrm{Cl}_{2}$ 10:1, column chromatography (EtOAc-MeOH 2:1) gave 35 (157 $\mathrm{mg}, 96 \%)$ as an orange solid: M. p. $74{ }^{\circ} \mathrm{C}$ (decomposition); $[\alpha]_{\mathrm{D}}+4^{\circ}(c 0.25, \mathrm{MeOH})$; $\mathrm{IR}\left(\mathrm{KBr}, \mathrm{cm}^{-1}\right)$ : 3397, 2919, 1067, 1040; ${ }^{1} \mathrm{H}-\mathrm{NMR}\left(300 \mathrm{MHz}, \mathrm{D}_{2} \mathrm{O}, 60^{\circ} \mathrm{C}\right.$ ), $\delta$ (ppm): 8.45 (s, $1 \mathrm{H}, \mathrm{H}-5-\mathrm{C}_{2} \mathrm{HN}_{3}$ ), 5.20 (bs, $\left.2 \mathrm{H}, \mathrm{H}_{\mathrm{Cp}}\right), 5.03\left(\mathrm{t}, 2 \mathrm{H},{ }^{3} J=5.1 \mathrm{~Hz}, \mathrm{CH}_{2} \mathrm{~N}\right), 4.84\left(\mathrm{bs}, 2 \mathrm{H}, \mathrm{H}_{\mathrm{Cp}}\right), 4.80\left(\mathrm{~d}, 1 \mathrm{H},{ }^{3} J_{1,2}=8.0 \mathrm{~Hz}, \mathrm{H}-1\right), 4.65(\mathrm{dd}$, $\left.1 \mathrm{H},{ }^{2} J=10.9 \mathrm{~Hz},{ }^{3} J=5.1 \mathrm{~Hz}, \mathrm{CH}_{2} \mathrm{O}\right), 4.56\left(\mathrm{~s}, 5 \mathrm{H}, \mathrm{H}_{\mathrm{Cp}}\right), 4.50\left(\mathrm{dd}, 1 \mathrm{H},{ }^{2} J=11.3 \mathrm{~Hz},{ }^{3} J=5.6 \mathrm{~Hz}, \mathrm{CH}_{2} \mathrm{O}\right.$ ), $4.25\left(\mathrm{dd}, 1 \mathrm{H},{ }^{2} J_{6,6}=12.2 \mathrm{~Hz},{ }^{3} J_{5,6}=2.1 \mathrm{~Hz}, \mathrm{H}-6\right), 4.06\left(\mathrm{dd}, 1 \mathrm{H},{ }^{2} J_{6,6},=12.2 \mathrm{~Hz},{ }^{3} J_{5,6}=5.6 \mathrm{~Hz}, \mathrm{H}-6\right.$ '), $3.84\left(\mathrm{t}, 1 \mathrm{H},{ }^{3} \mathrm{~J}=8.9 \mathrm{~Hz}, \mathrm{H}-3\right), 5.77\left(\mathrm{dd}, 1 \mathrm{H},{ }^{3} J_{5,6}=5.6 \mathrm{~Hz},{ }^{3} J_{5,6}=2.3 \mathrm{~Hz}, \mathrm{H}-5\right), 3.73\left(\mathrm{dd}, 1 \mathrm{H},{ }^{3} J_{3,4}=8.4\right.$ $\left.\mathrm{Hz},{ }^{3} J_{4,5}=1.8 \mathrm{~Hz}, \mathrm{H}-4\right), 3.64\left(\mathrm{t}, 1 \mathrm{H},{ }^{3} J=8.5 \mathrm{~Hz}, \mathrm{H}-2\right) ;{ }^{13} \mathrm{C}-\mathrm{NMR}\left(75 \mathrm{MHz}, \mathrm{D}_{2} \mathrm{O}, 60{ }^{\circ} \mathrm{C}\right), \delta(\mathrm{ppm}): 147.2$ $\left(\mathrm{C}-4-\mathrm{C}_{2} \mathrm{HN}_{3}\right), 122.4\left(\mathrm{C}-5-\mathrm{C}_{2} \mathrm{HN}_{3}\right), 102.8(\mathrm{C}-1), 76.4(\mathrm{C}-5), 76.2$ (C-3), $74.8\left(\mathrm{C}_{\mathrm{ipso}}\right), 73.5(\mathrm{C}-2), 70.4$ $\left(\mathrm{C}_{\mathrm{Cp}^{p}}\right), 70.2(\mathrm{C}-4), 69.8\left(\mathrm{C}_{\mathrm{Cp}_{\mathrm{p}}}\right), 68.2\left(\mathrm{CH}_{2} \mathrm{O}\right), 67.2\left(\mathrm{C}_{\mathrm{Cp}}\right), 61.4(\mathrm{C}-6), 50.7\left(\mathrm{CH}_{2} \mathrm{~N}\right)$; HMRS (FAB+): Calc. for $\mathrm{C}_{20} \mathrm{H}_{25} \mathrm{O}_{6} \mathrm{~N}_{3} \mathrm{Fe}$ 459.1093. Found $482.0996[\mathrm{M}+\mathrm{Na}]^{+}$.

[1-( $\alpha$-D-Mannopyranosyloxyethyl-1H-1,2,3-triazol-4-yl]ferrocene (36). Starting from 30 (147 $\mathrm{mg}, 0.234 \mathrm{mmol}$ ) in dry $\mathrm{MeOH}$, column chromatography (EtOAc-MeOH 2:1) gave 36 (102 mg, 95\%) as an orange solid: M. p. $66^{\circ} \mathrm{C}$ (decomposition); $[\alpha]_{\mathrm{D}}+26^{\circ}(c 0.25, \mathrm{MeOH}) ; \mathrm{IR}\left(\mathrm{KBr}, \mathrm{cm}^{-1}\right): 3404,2923$, 1053; ${ }^{1} \mathrm{H}-\mathrm{NMR}\left(300 \mathrm{MHz}, \mathrm{CD}_{3} \mathrm{OD}\right), \delta(\mathrm{ppm}): 8.02$ (s, $\left.1 \mathrm{H}, \mathrm{H}-5-\mathrm{C}_{2} \mathrm{HN}_{3}\right), 4.76\left(\mathrm{~d}, 1 \mathrm{H},{ }^{3} J_{1,2}=1.4 \mathrm{~Hz}, \mathrm{H}-1\right)$, $4.74\left(\mathrm{t}, 2 \mathrm{H},{ }^{3} J=1.7 \mathrm{~Hz}, \mathrm{H}_{\mathrm{Cp}}\right), 4.66-4.61\left(\mathrm{~m}, 2 \mathrm{H} \mathrm{CH}_{2} \mathrm{~N}\right), 4.32\left(\mathrm{t}, 2 \mathrm{H},{ }^{3} J=1.7 \mathrm{~Hz}, \mathrm{H}_{\mathrm{Cp}}\right), 4.17$ (ddd, $1 \mathrm{H},{ }^{2} J=$ $10.4 \mathrm{~Hz},{ }^{3} J=5.9 \mathrm{~Hz},{ }^{3} J=4.5 \mathrm{~Hz}, \mathrm{CH}_{2} \mathrm{O}$ ), $4.07\left(\mathrm{~s}, 5 \mathrm{H}, \mathrm{H}_{\mathrm{Cp}}\right.$ ), $3.92\left(\mathrm{ddd}, 1 \mathrm{H},{ }^{2} J=10.7 \mathrm{~Hz},{ }^{3} J=6.4 \mathrm{~Hz},{ }^{3} J\right.$ $\left.=4.5 \mathrm{~Hz}, \mathrm{CH}_{2} \mathrm{O}\right), 3.82\left(\mathrm{dd}, 1 \mathrm{H},{ }^{2} J_{6,6}=11.7 \mathrm{~Hz},{ }^{3} J_{5,6}=2.4 \mathrm{~Hz}, \mathrm{H}-6\right) 3.77\left(\mathrm{dd}, 1 \mathrm{H},{ }^{3} J_{1,2}=1.7 \mathrm{~Hz},{ }^{3} J_{2,3}=\right.$ $3.0 \mathrm{~Hz}, \mathrm{H}-2), 3.68\left(\mathrm{dd}, 1 \mathrm{H},{ }^{2} J_{6,6}=11.8 \mathrm{~Hz},{ }^{3} J_{5,6}=6.0 \mathrm{~Hz}, \mathrm{H}-6\right.$ ) $) 3.65\left(\mathrm{dd}, 1 \mathrm{H},{ }^{3} J_{3,4}=9.2 \mathrm{~Hz},{ }^{3} J_{2,3}=3.2\right.$ $\mathrm{Hz}, \mathrm{H}-3$ ), 3.59 (t, $\left.1 \mathrm{H},{ }^{3} J=9.3 \mathrm{~Hz}, \mathrm{H}-4\right), 3.39$ (ddd, $1 \mathrm{H},{ }^{3} J_{4,5}=8.6 \mathrm{~Hz},{ }^{3} J_{5,6}=5.9 \mathrm{~Hz},{ }^{3} J_{5,6}=2.1 \mathrm{~Hz}, \mathrm{H}-5$ ); ${ }^{13} \mathrm{C}-\mathrm{NMR}\left(75 \mathrm{MHz}, \mathrm{CD}_{3} \mathrm{OD}\right), \delta(\mathrm{ppm}): 148.0\left(\mathrm{C}-4-\mathrm{C}_{2} \mathrm{HN}_{3}\right), 122.0\left(\mathrm{C}-5-\mathrm{C}_{2} \mathrm{HN}_{3}\right), 101.7(\mathrm{C}-1), 76.1\left(\mathrm{C}_{\mathrm{ips}}\right)$, $75.0(\mathrm{C}-5), 72.5(\mathrm{C}-3), 71.9(\mathrm{C}-2), 70.6\left(\mathrm{C}_{\mathrm{Cp}}\right), 69.8\left(\mathrm{C}_{\mathrm{Cp}}\right), 68.3(\mathrm{C}-4), 67.7\left(\mathrm{C}_{\mathrm{Cp}_{\mathrm{p}}}\right), 66.8\left(\mathrm{CH}_{2} \mathrm{O}\right), 62.8(\mathrm{C}-$ 6), $51.2\left(\mathrm{CH}_{2} \mathrm{~N}\right)$; HMRS (FAB+): Calc. for $\mathrm{C}_{20} \mathrm{H}_{25} \mathrm{O}_{6} \mathrm{~N}_{3} \mathrm{Fe} 459.1093$. Found $482.0993[\mathrm{M}+\mathrm{Na}]^{+}$. 


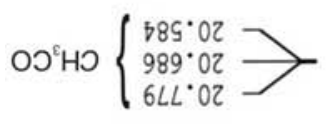

$S^{2} \mathrm{HO} 255^{\circ} 62$

고은

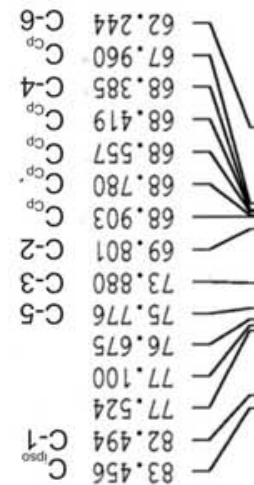

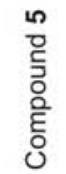
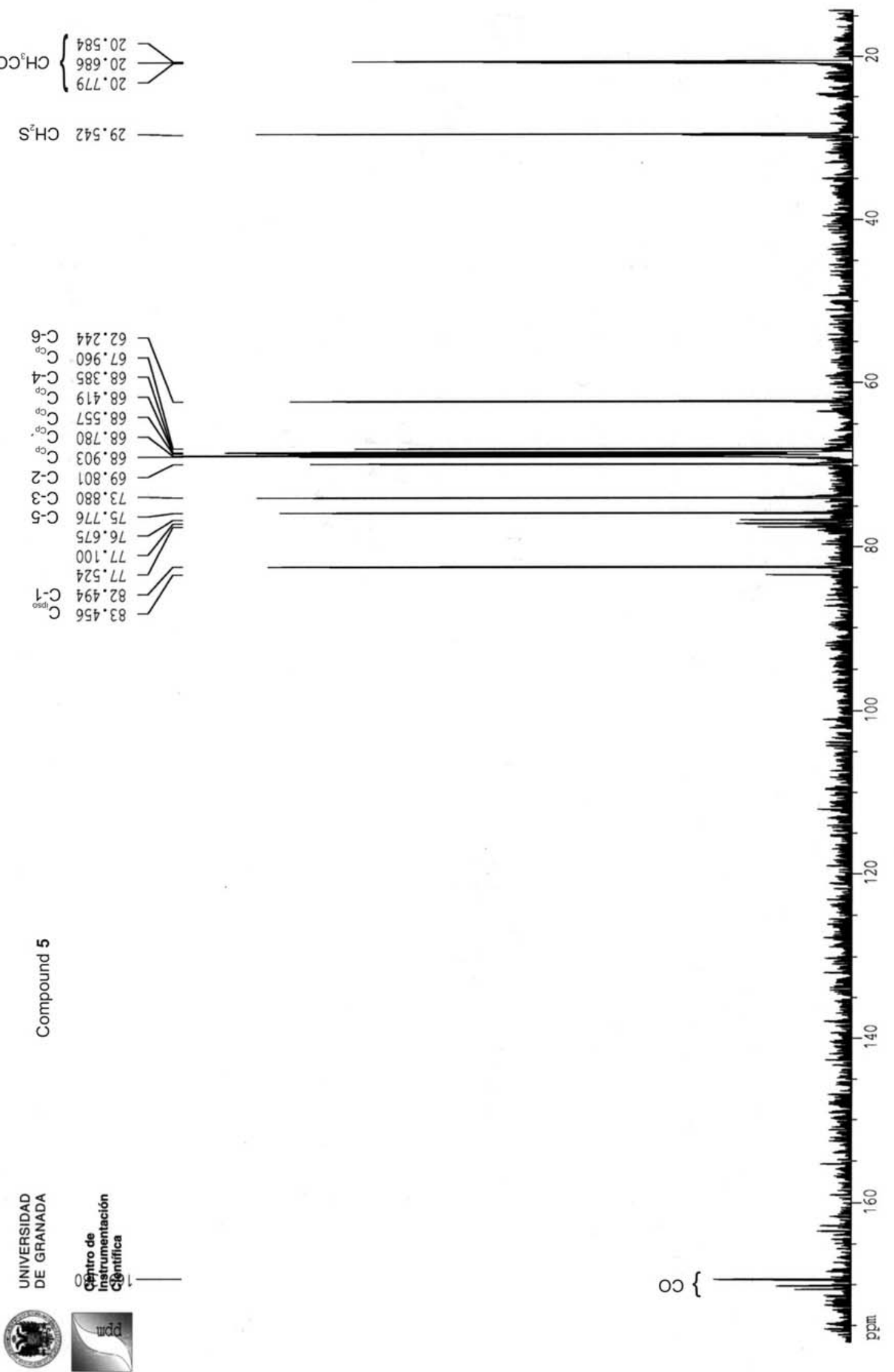

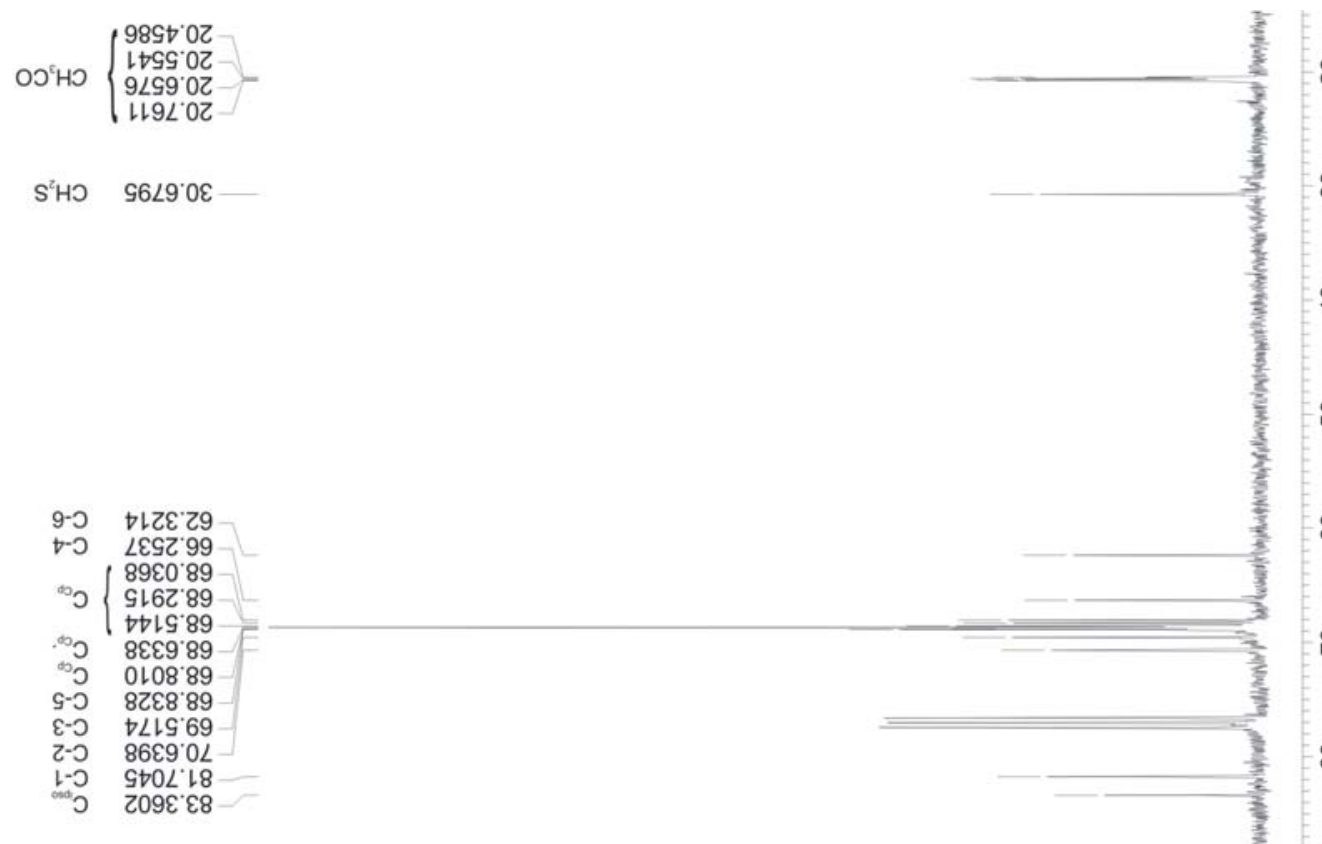

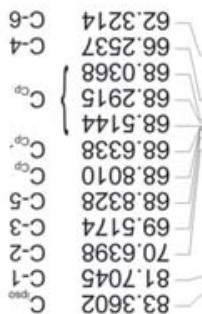




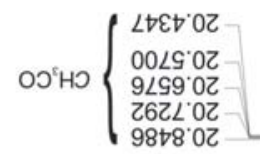

SHO $8029^{\circ} 62$

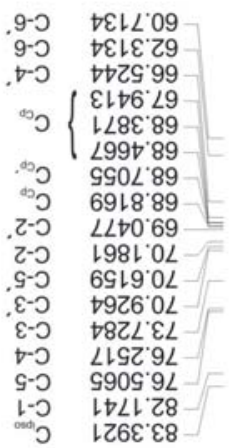

. - $6 \varepsilon Z 0$ LOL

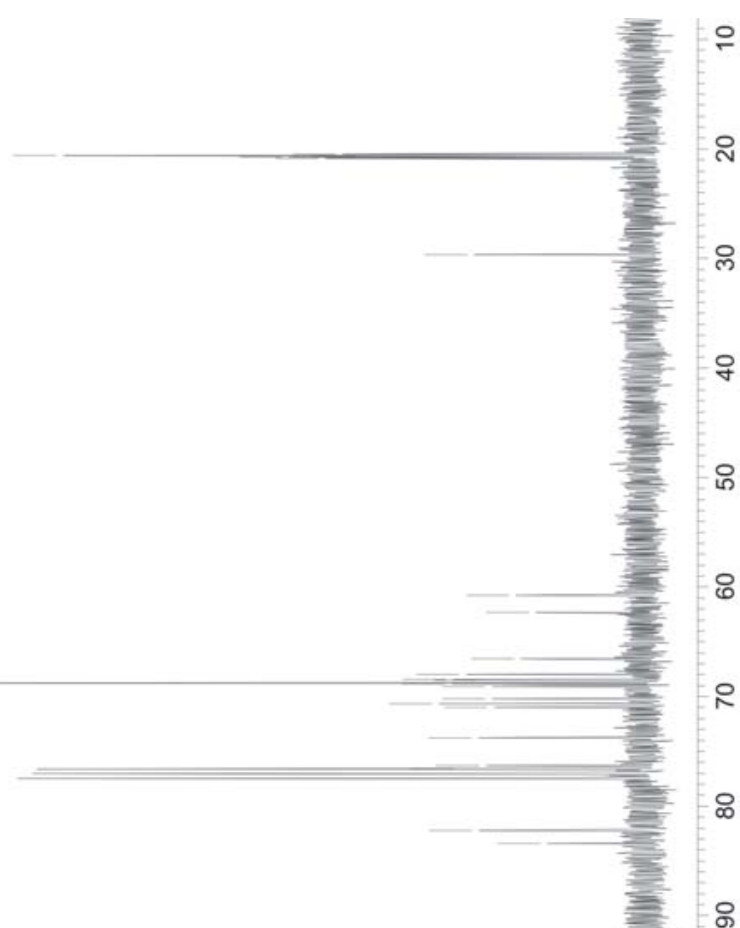

ㅇ

흥

웅

울

ํำ

윰

g

요

$\stackrel{\circ}{\circ}$

온 

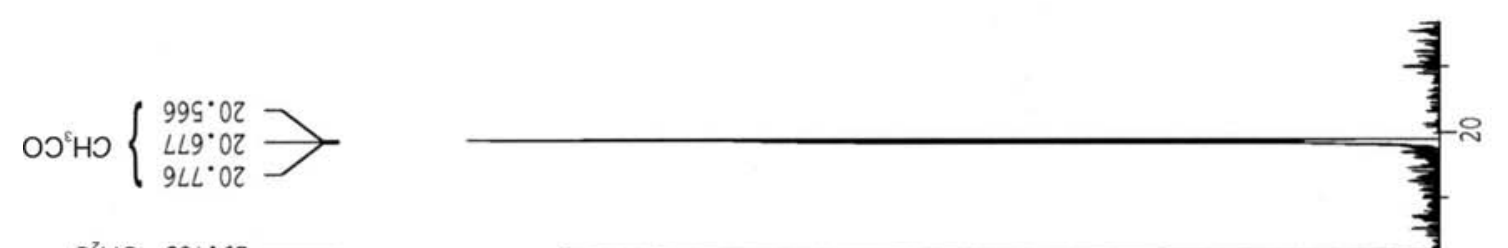

$S^{2} H O 581 \cdot 62$
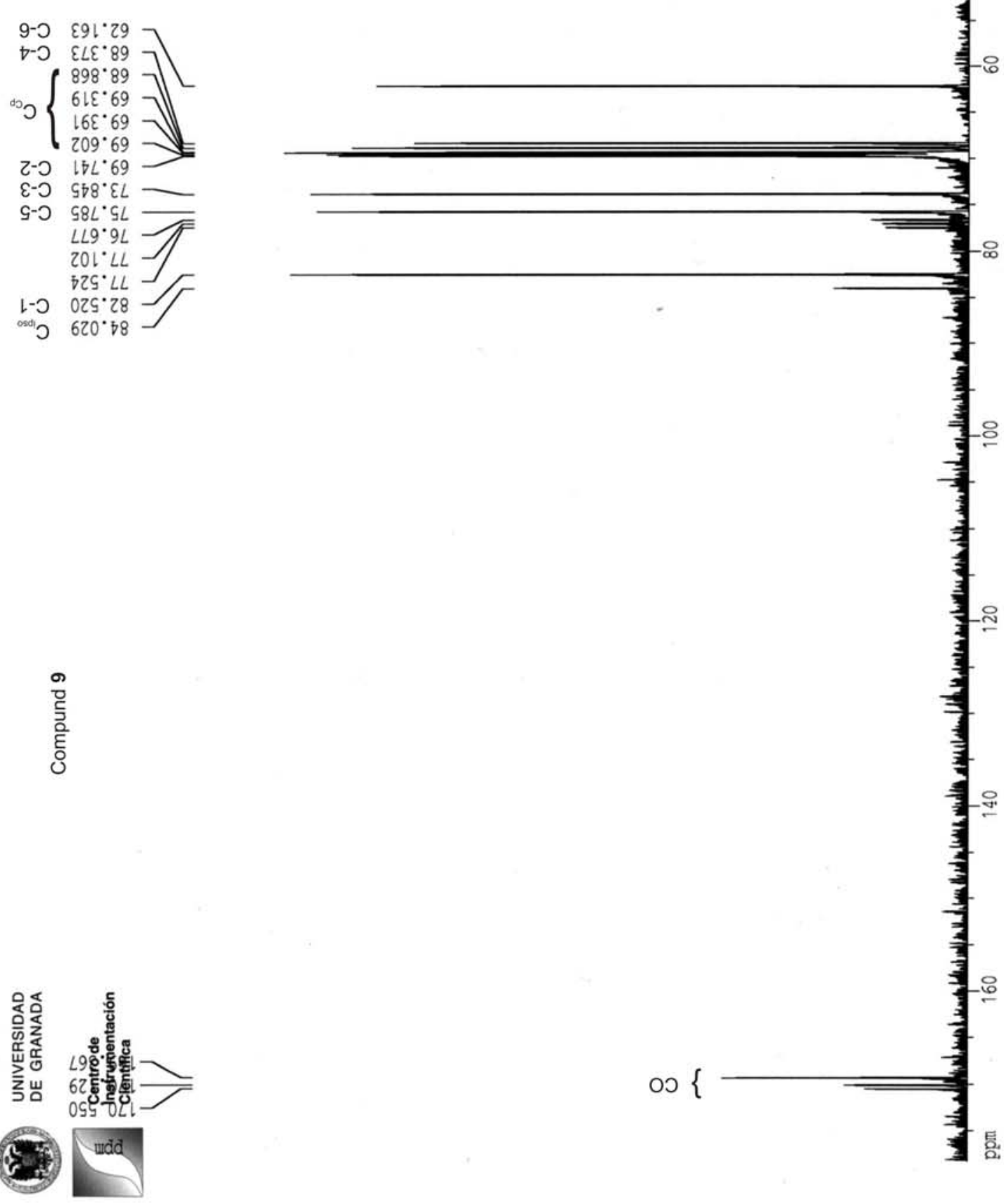


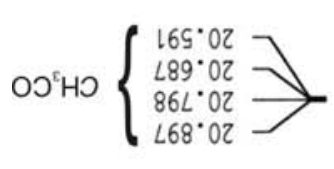

$\mathrm{S}^{2} \mathrm{HO} \quad 699^{\circ} \mathrm{OE}-$
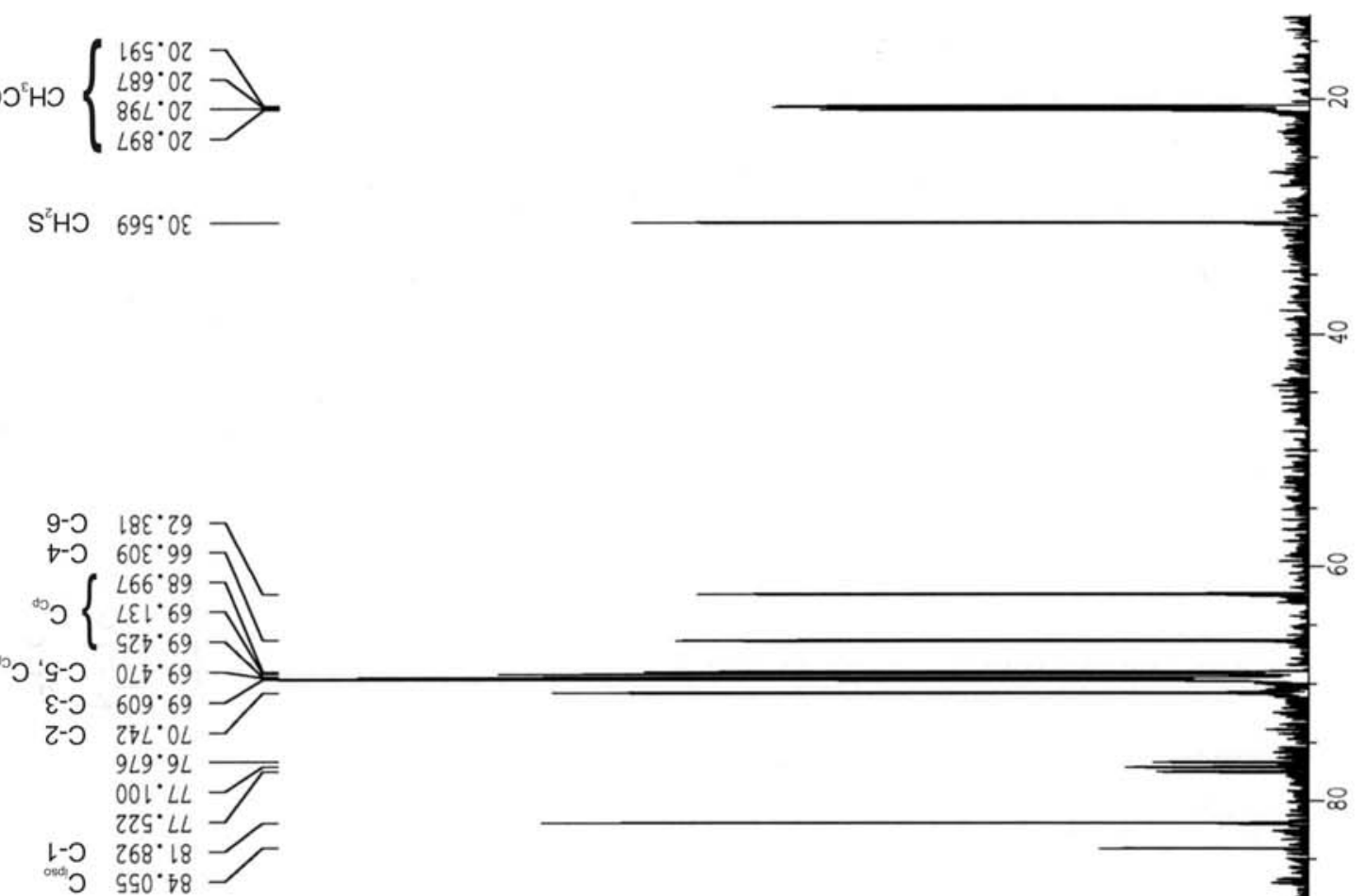

울
형
흥

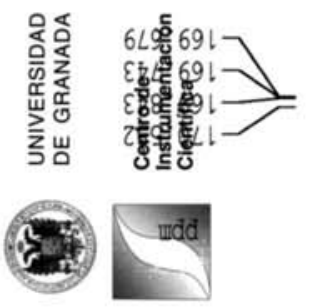

०ว
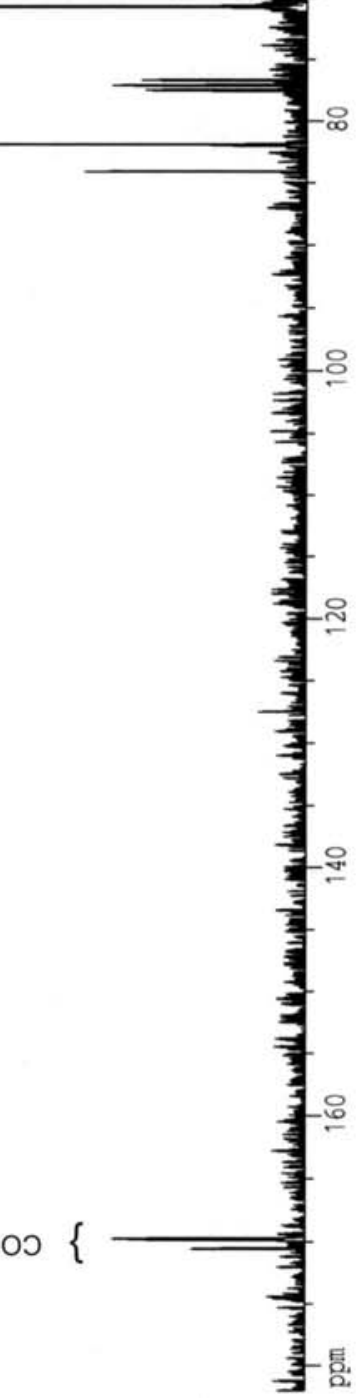


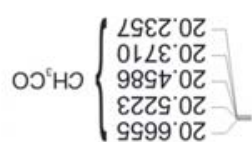

$S^{2}$ HO $9890^{\circ} 62$

d.-2 $18 \angle \mathrm{g} .09$

t-5 6ZLt 99

8929.89

9888 89

S $\angle 8069$

z-o tole 69

s-5 680t02 02

E-ว

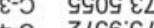

S-ว ZSSE $9 L$

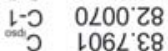

L-5 LS8L'00L

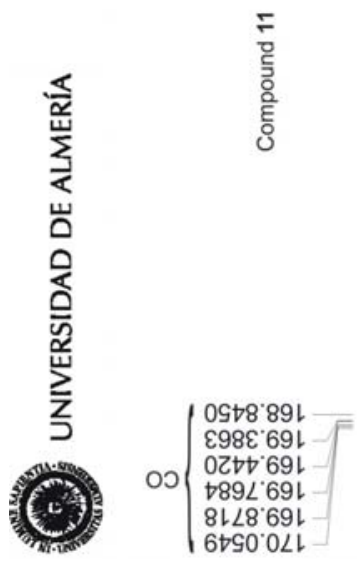


$S^{2}$ HO ZSเ6 62

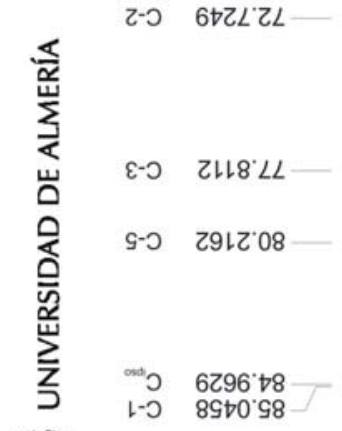




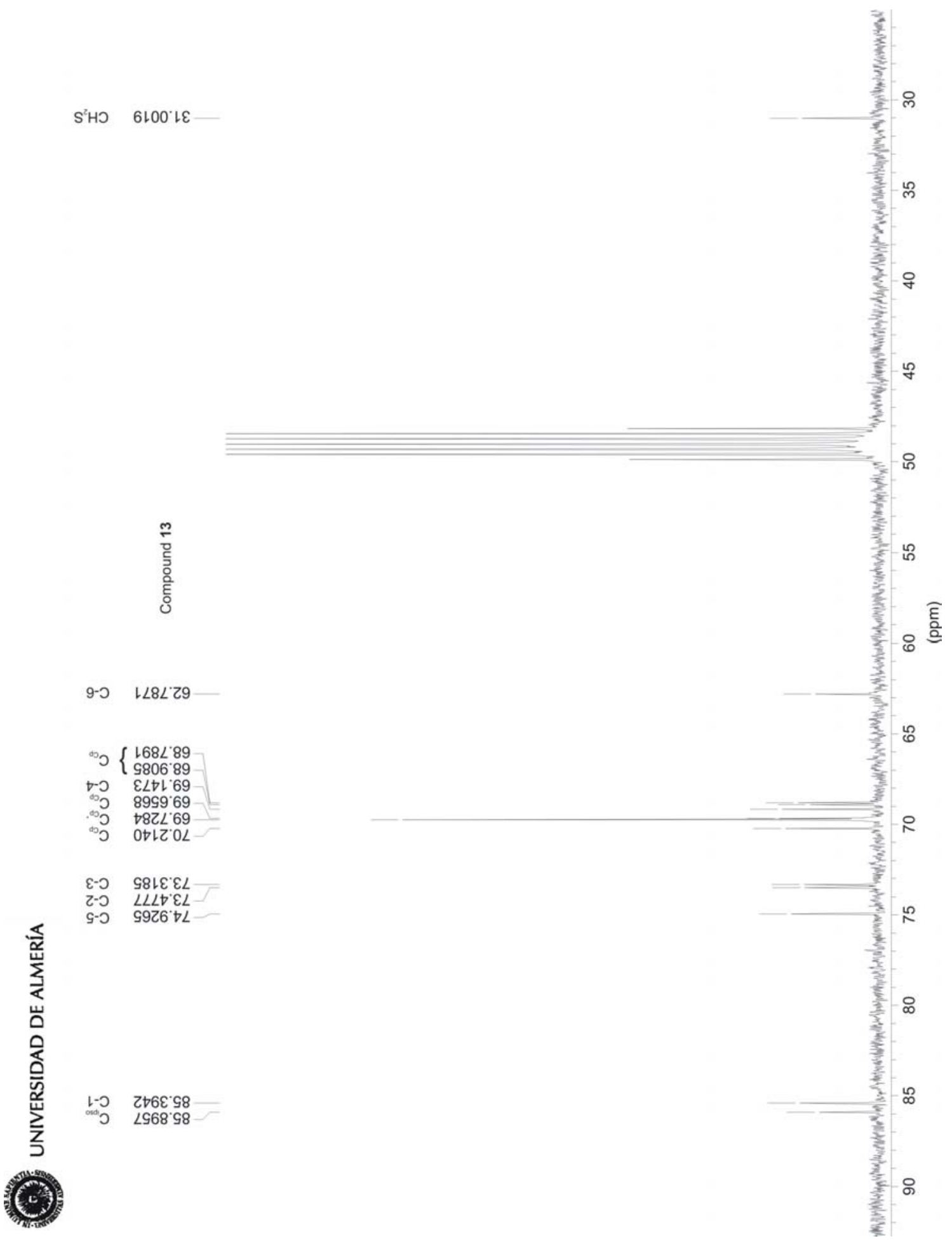


t $=0$ 888Z $0 L$

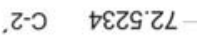

$z-0 \quad \varepsilon t 90 \circ t$

. $\varepsilon=0$. $6 \varepsilon 6 L ' t L$

$\begin{array}{ll}. \varsigma 5 & 0 L L 0^{\circ} L L \\ \varepsilon-5 & 9086: L L\end{array}$

$\begin{array}{ll}\mathrm{s}-5 & \text { เttb' } 08 \\ \mathrm{t}-5 & 88 t \mathrm{G}^{\circ} 08\end{array}$

$\begin{array}{cc}5 & \text { sLL } \\ \iota-0 & 8 Z 28.98\end{array}$

交 
$S^{2} H O \quad 80 Z L^{\circ} 6 Z$

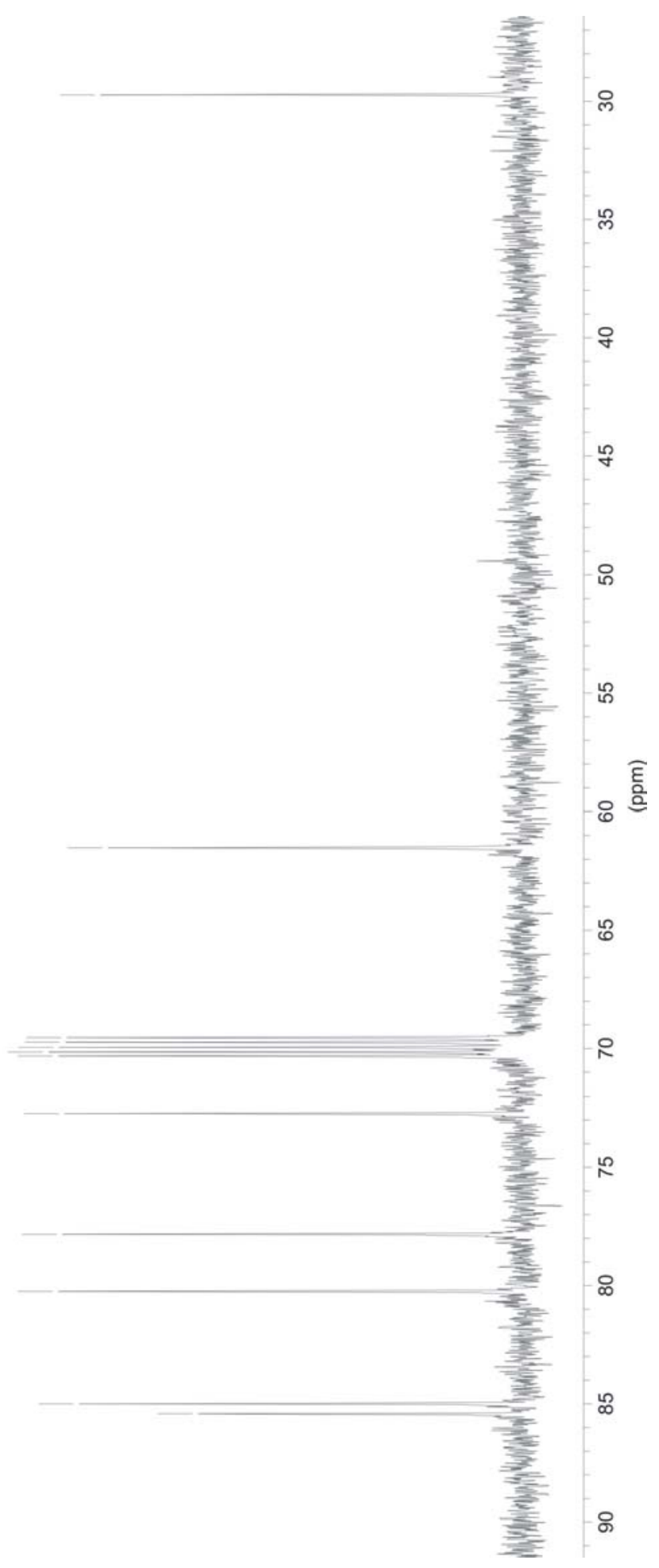




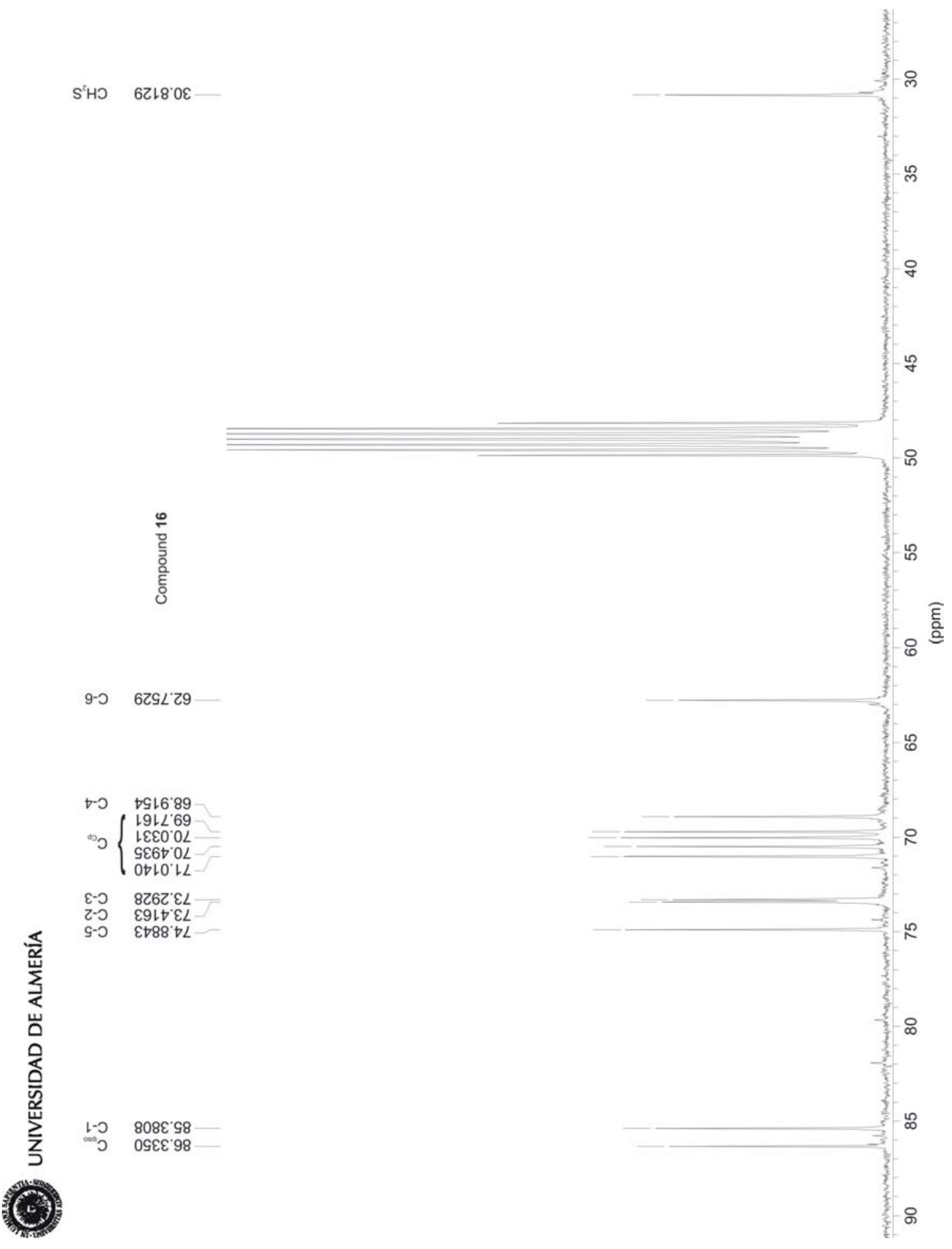


$S^{2} H O \quad 8 S+6: L 2$

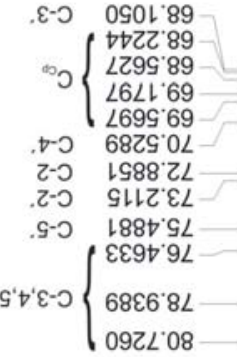



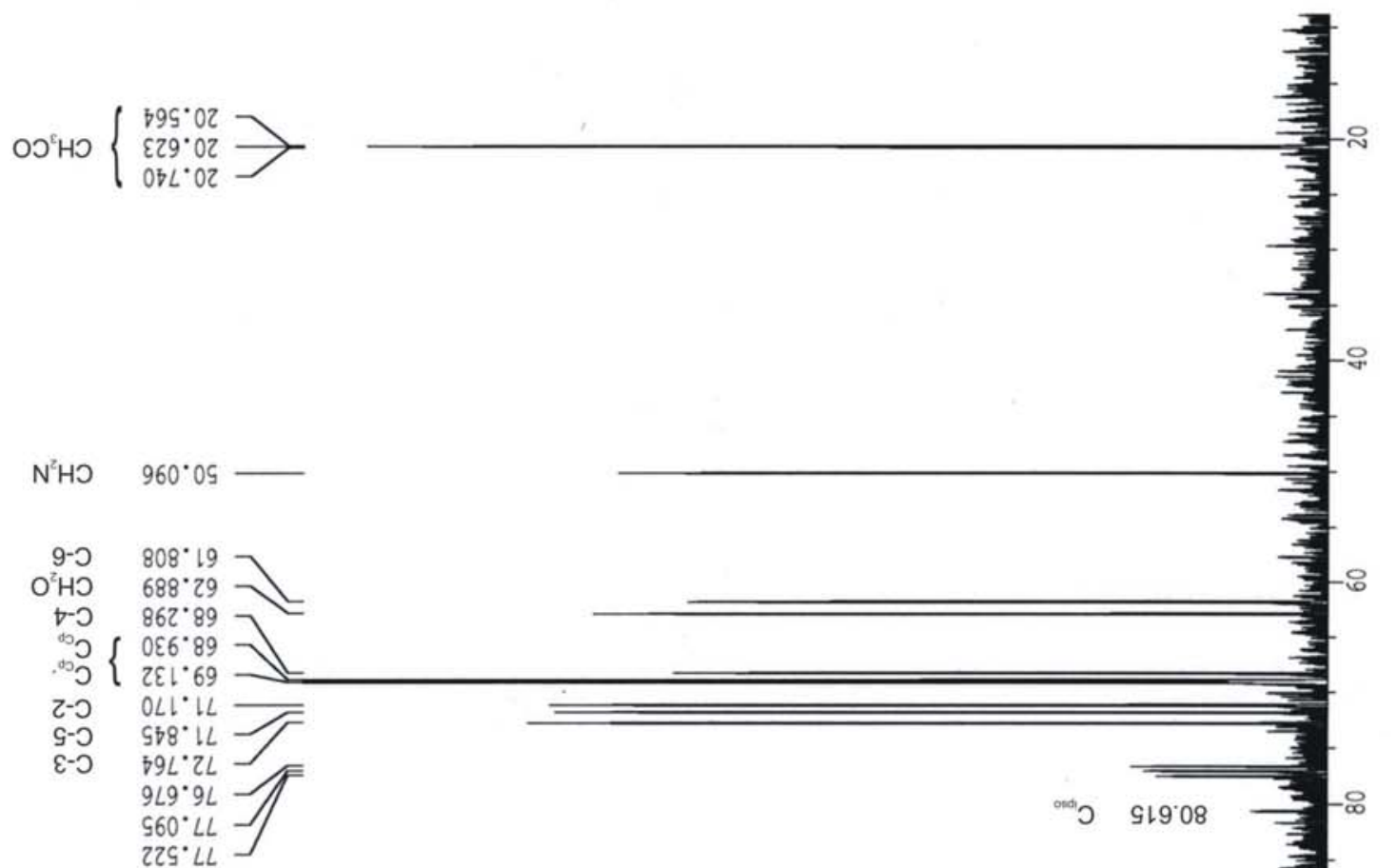

L-O $\quad b ! L^{*} 66-$

${ }^{\mathrm{NH}} \mathrm{H}^{2} \mathrm{O}-\mathrm{S}-\mathrm{O} \quad 1 / \mathrm{O}^{\circ} 221-$<smiles>O=[SH]c1ccccc1</smiles>

$\mathrm{NH}^{2} \mathrm{O}-\nabla-0 \quad \nabla 20+\nabla t$

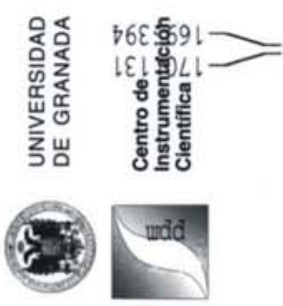

००
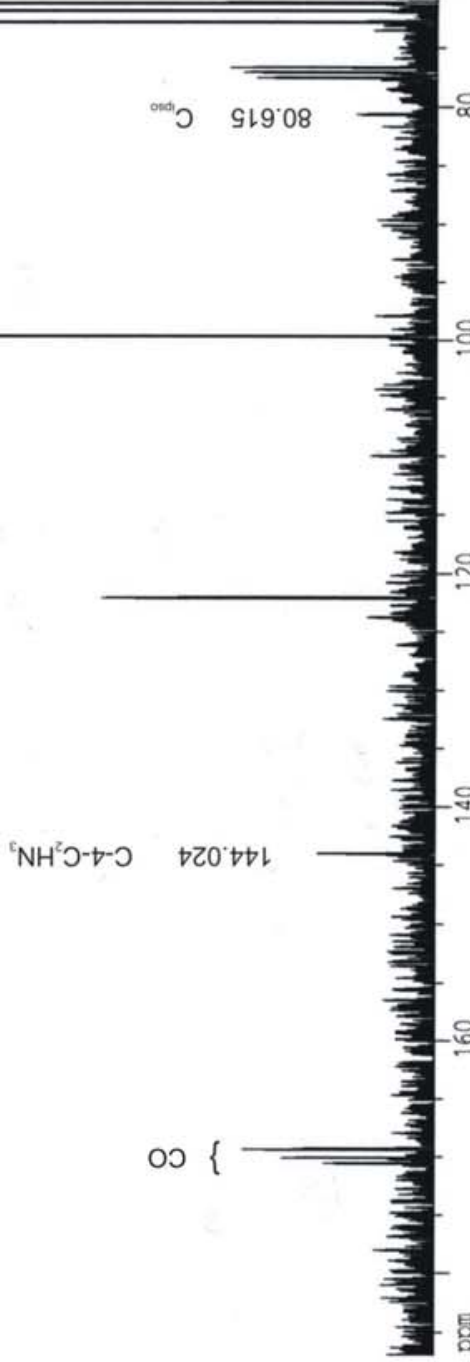


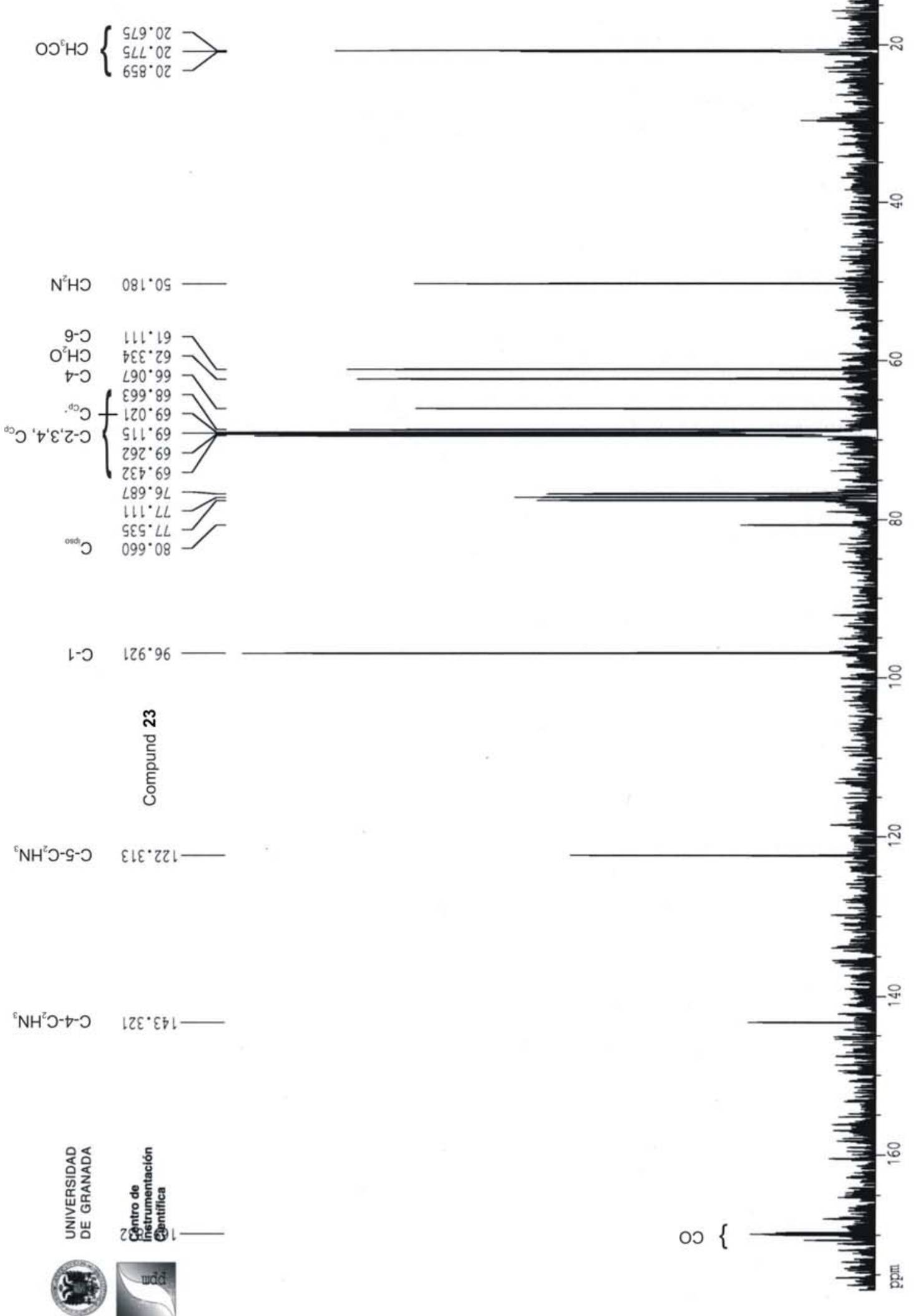




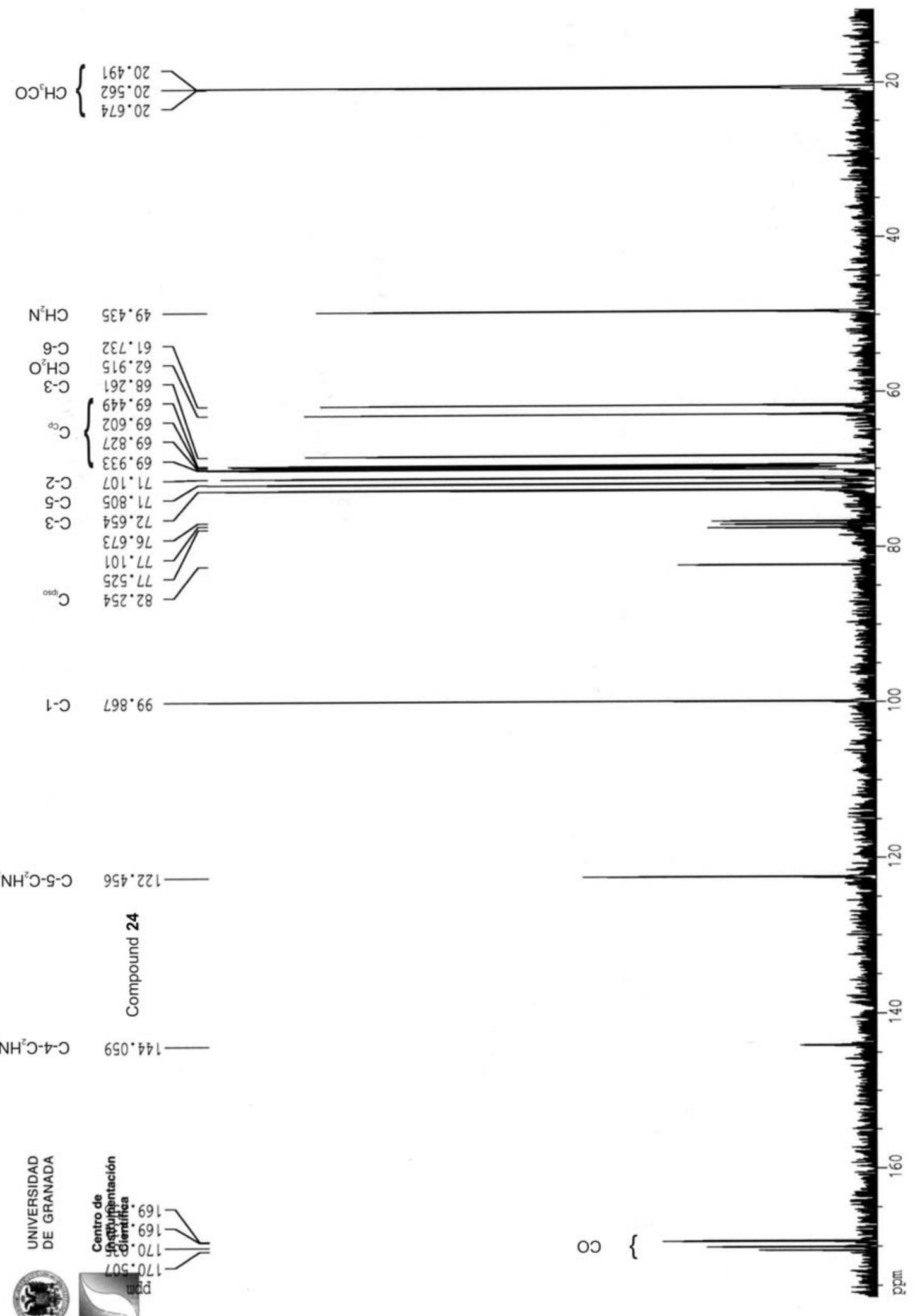



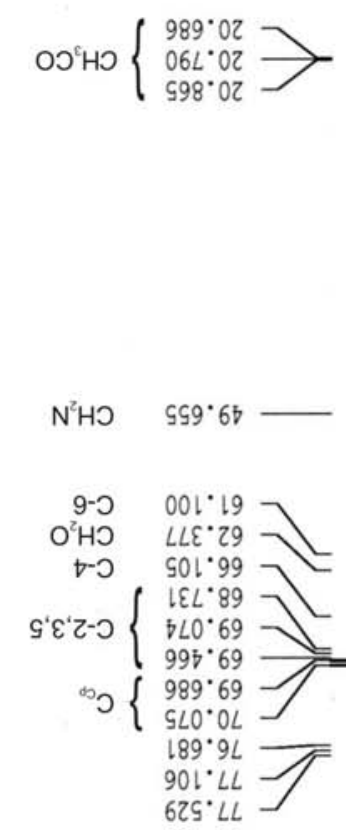

เ-ว $896.96-$

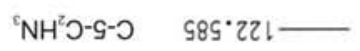

${ }^{\varepsilon} \mathrm{NH}^{2}$ כ-ち-ว

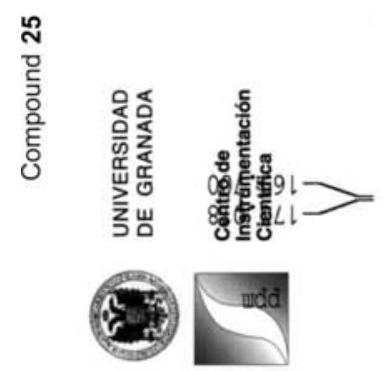




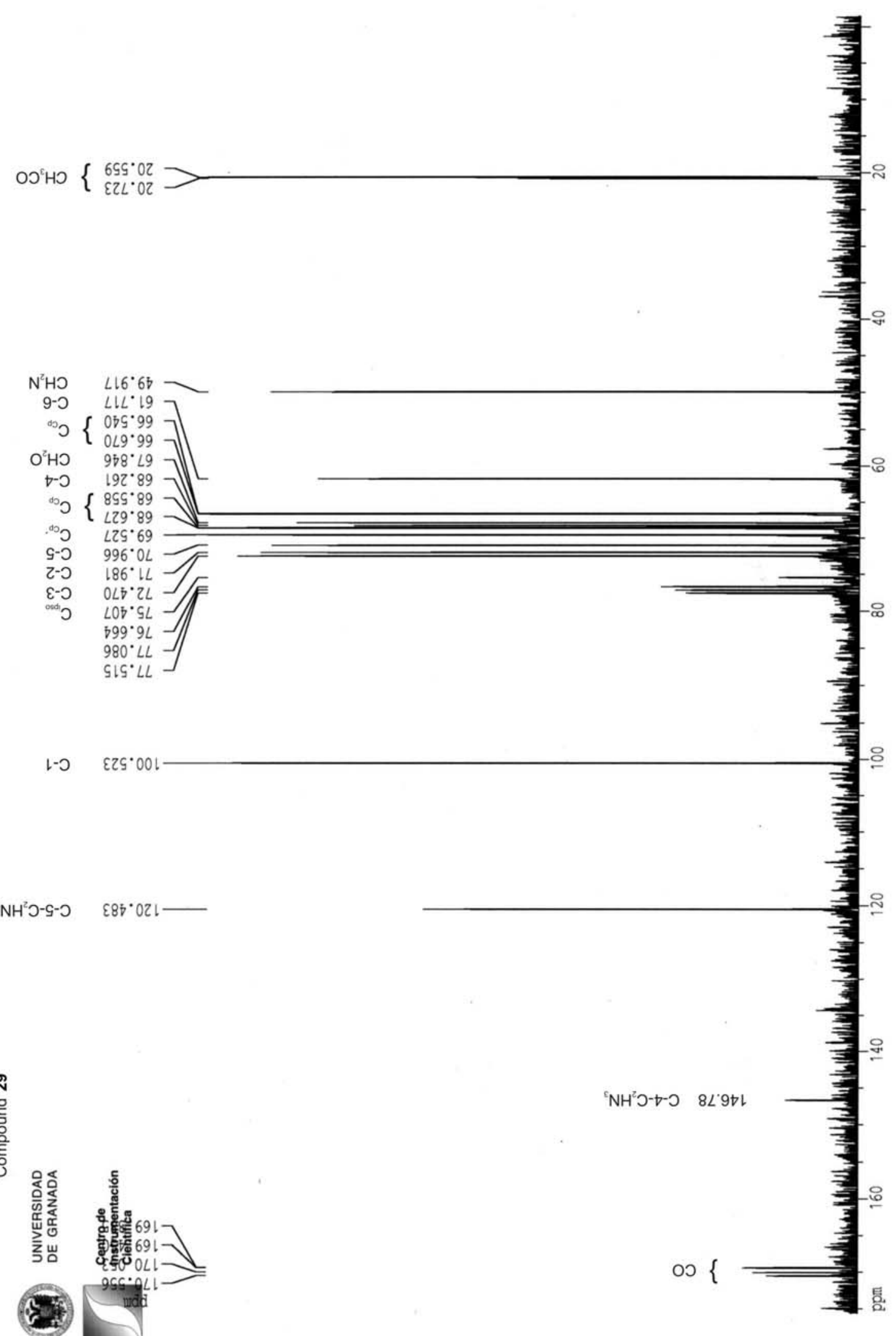



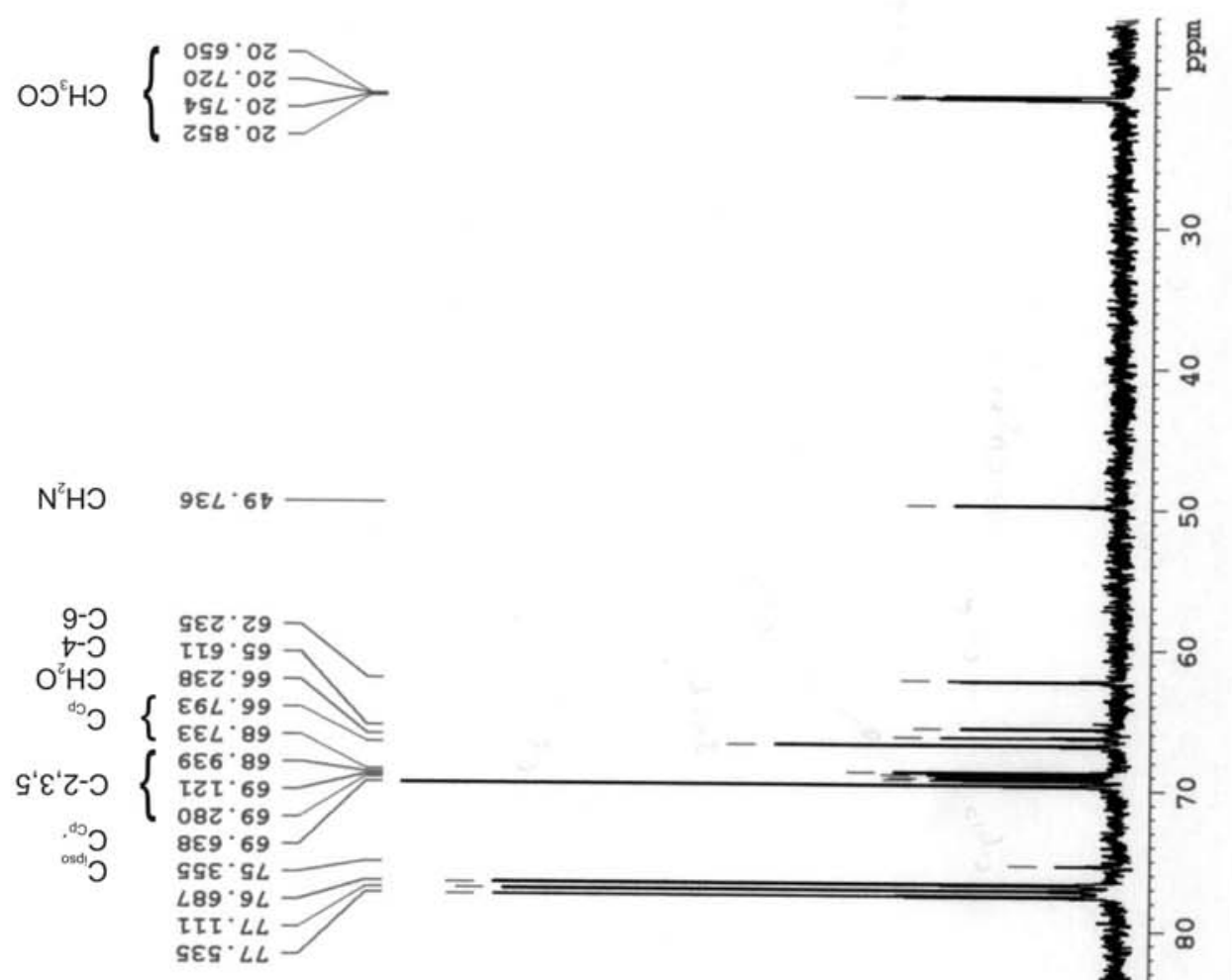

${ }^{\varepsilon} \mathrm{NH}^{2} \mathrm{O}-\mathrm{G}-\mathrm{O} \quad 8 \vee \tau \cdot 02 \tau$

${ }^{\varepsilon} \mathrm{NH}^{2} \mathrm{O}-\downarrow-0 \quad 8 \nabla \tau \cdot L \nabla \tau$

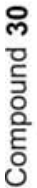
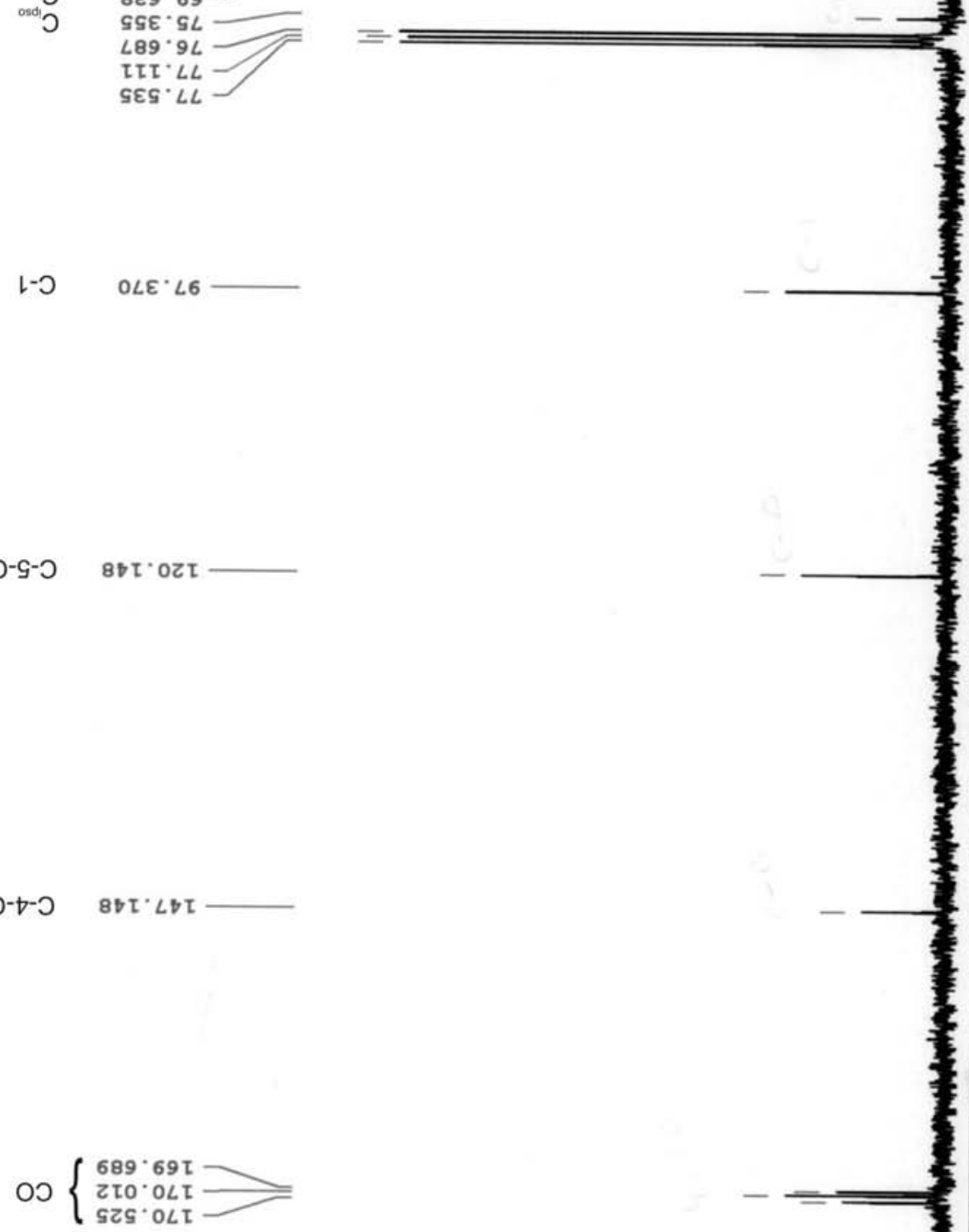

L-O OLE $\angle 6$

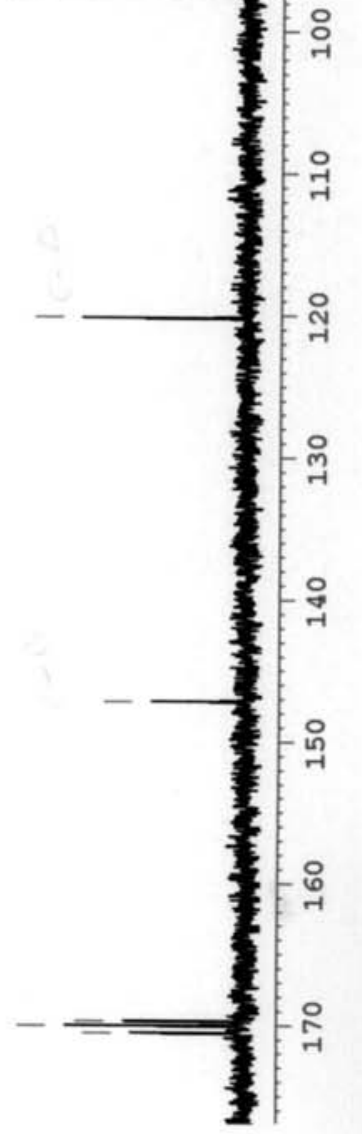




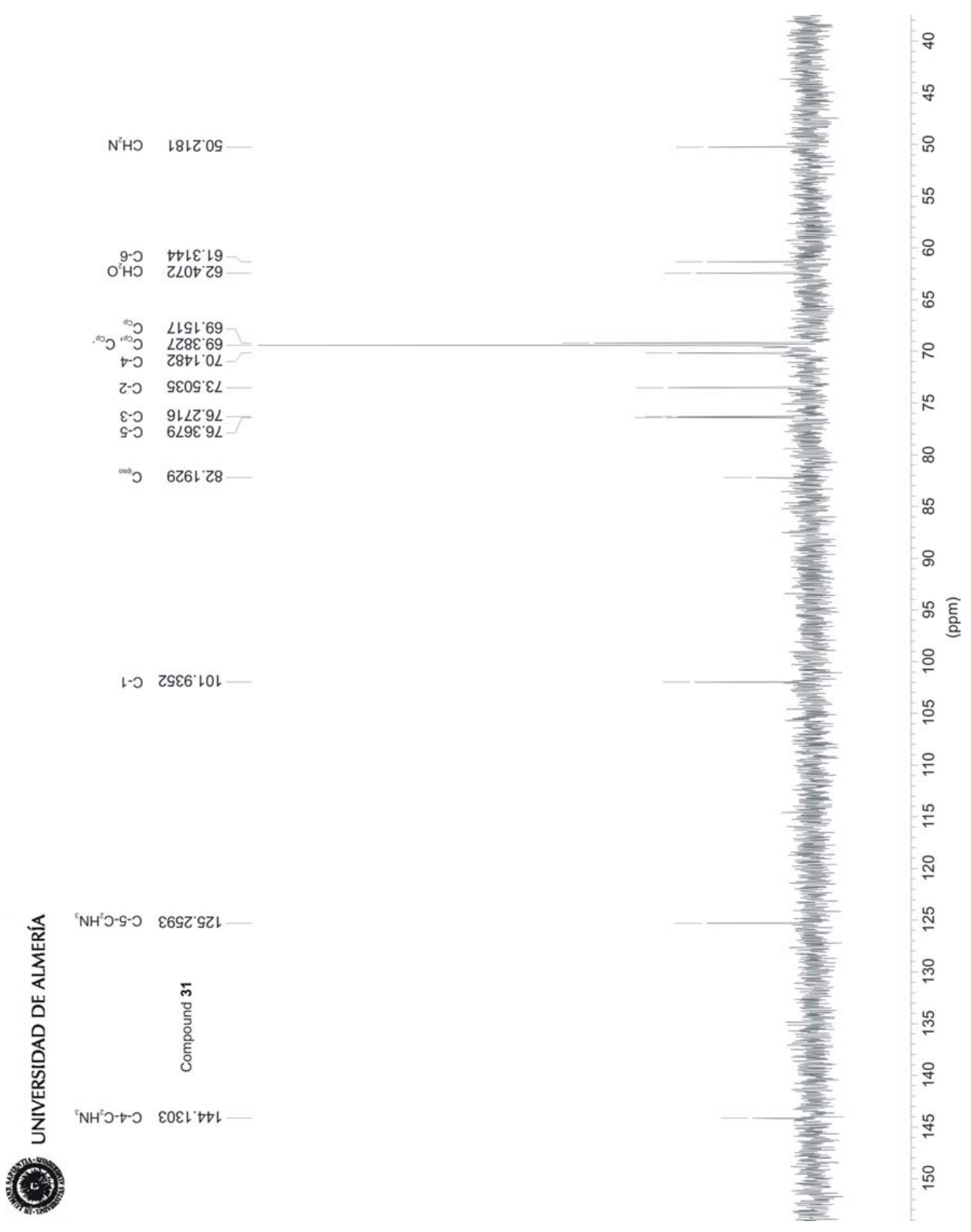




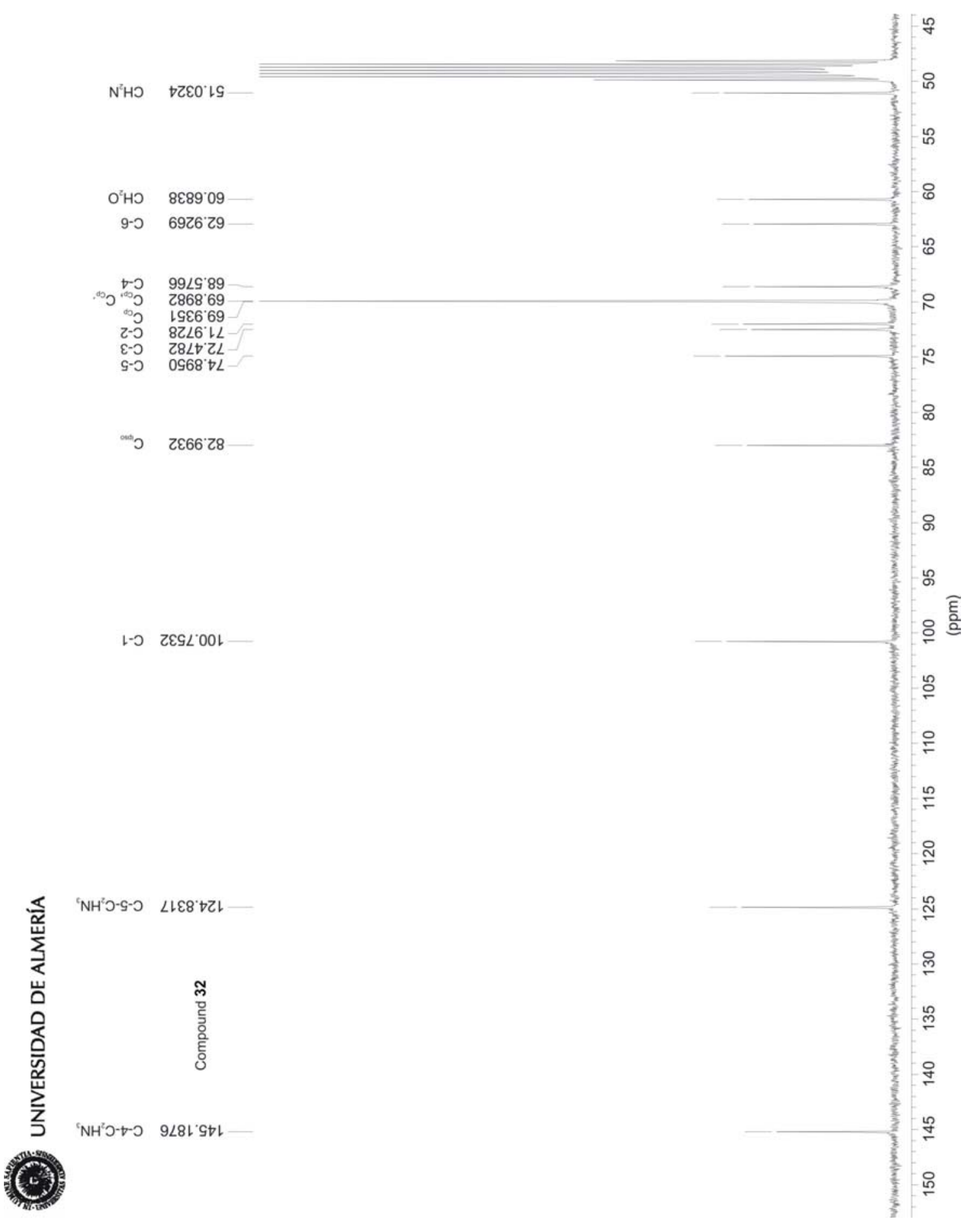




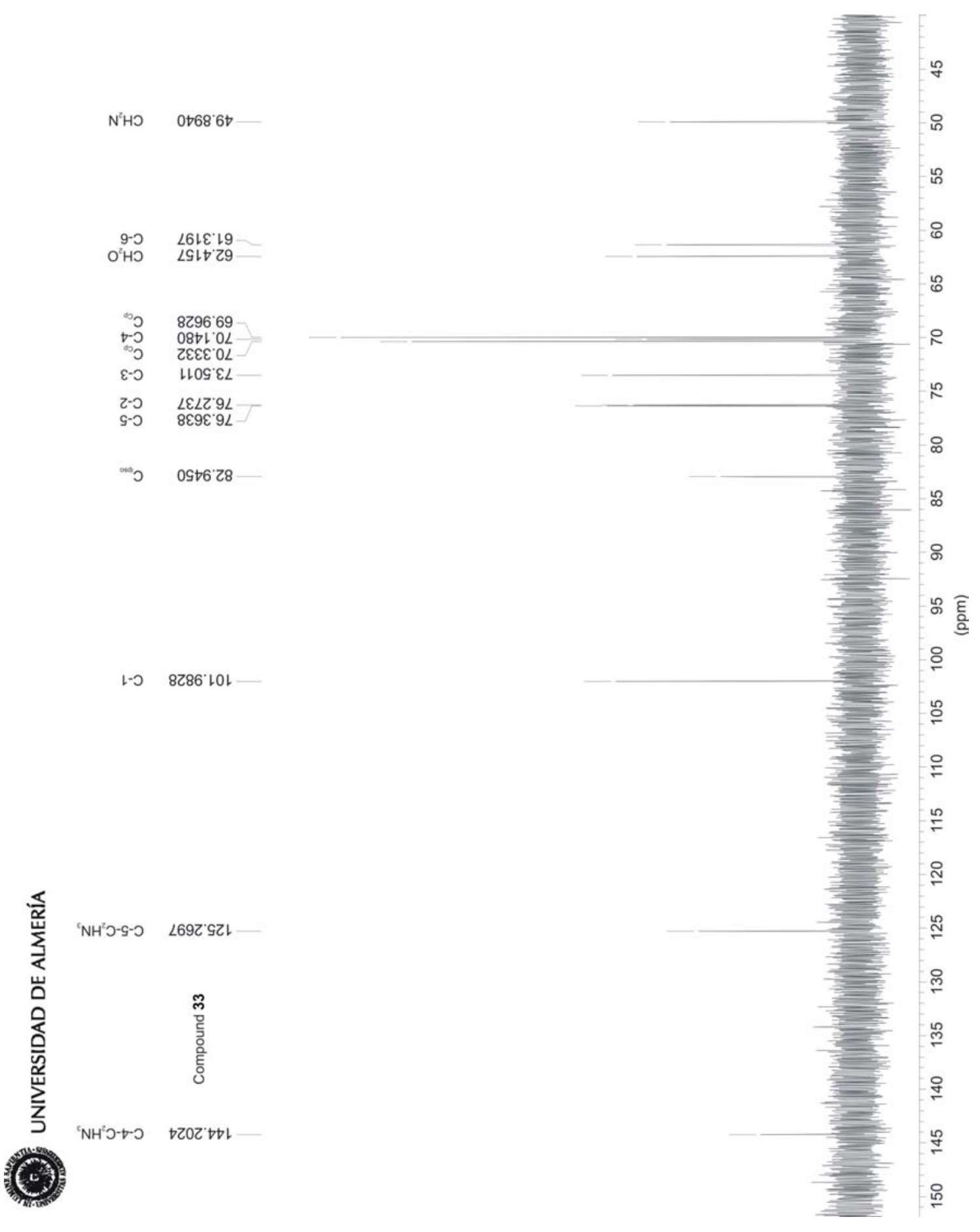




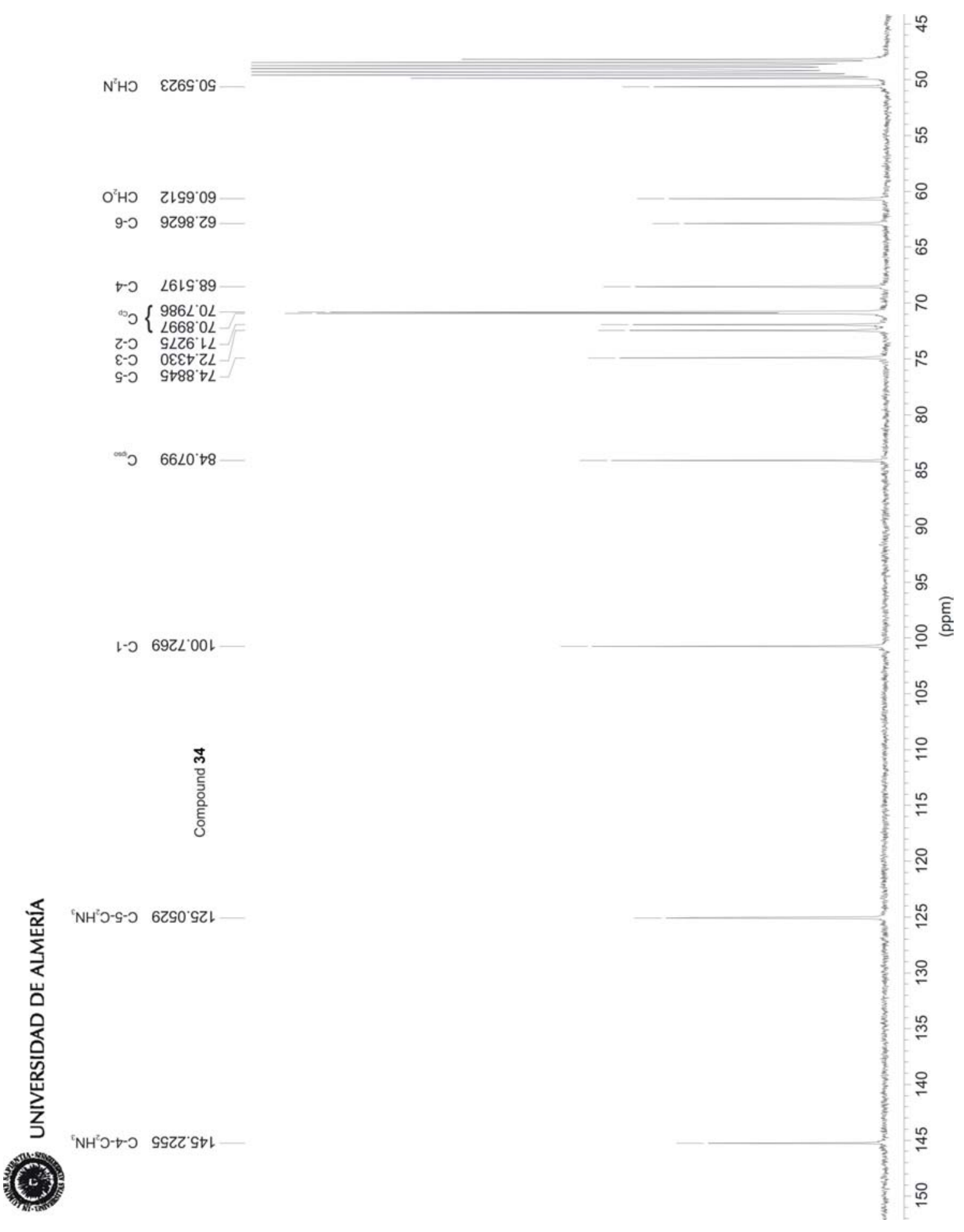




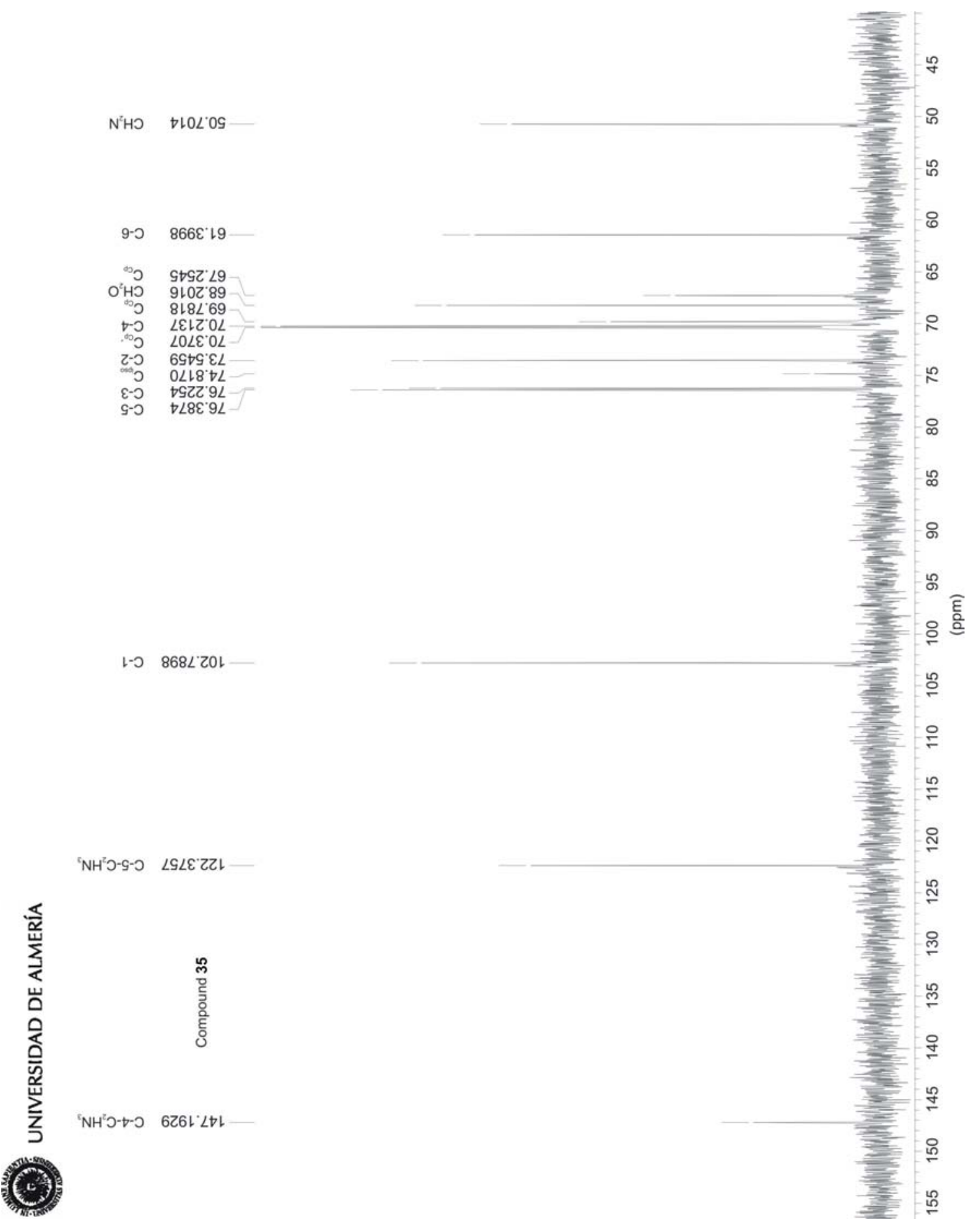




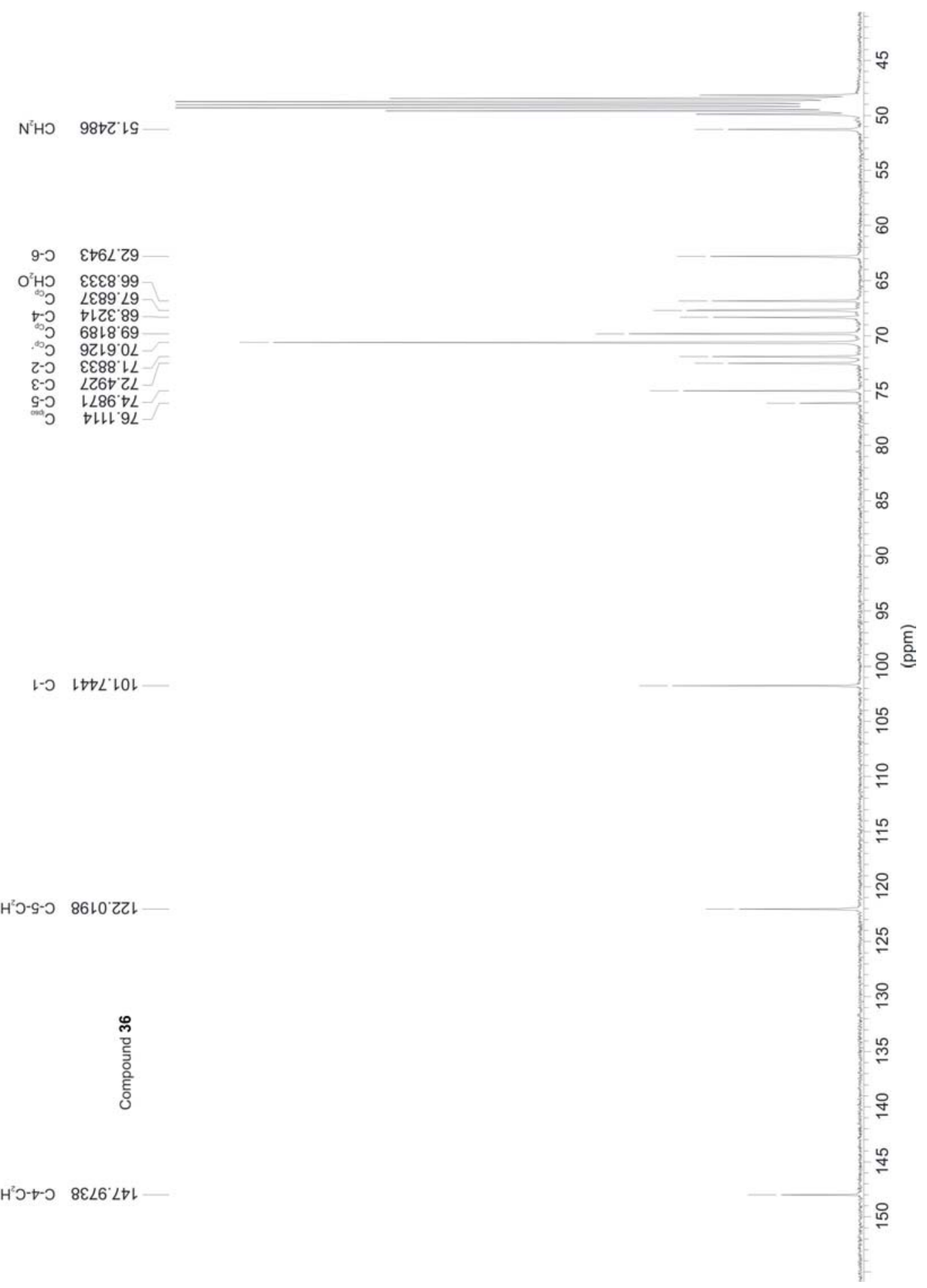

